\title{
Topological entanglement and hyperbolic volume
}

\author{
Aditya Dwivedi, ${ }^{a}$ Siddharth Dwivedi, ${ }^{b}$ Bhabani Prasad Mandal, ${ }^{a}$ Pichai Ramadevi ${ }^{c}$ \\ and Vivek Kumar Singh ${ }^{d}$ \\ ${ }^{a}$ Department of Physics, Institute of Science, Banaras Hindu University, \\ Varanasi, 221005, India \\ ${ }^{b}$ Center for Theoretical Physics, College of Physical Science and Technology, Sichuan University, \\ Chengdu, 610064, China \\ ${ }^{c}$ Department of Physics, Indian Institute of Technology Bombay, \\ Powai, Mumbai, 400076, India \\ ${ }^{d}$ Department of Mathematics, Indian Institute of Science Education and Research, Pune, \\ Pashan, Pune, 411008, India \\ E-mail: aditya.dwivedi13@bhu.ac.in, sdwivedi@scu.edu.cn, \\ bhabani@bhu.ac.in, ramadevi@phy.iitb.ac.in, vivek.singh@fuw.edu.pl
}

ABSTRACT: The entanglement entropy of many quantum systems is difficult to compute in general. They are obtained as a limiting case of the Rényi entropy of index $m$, which captures the higher moments of the reduced density matrix. In this work, we study pure bipartite states associated with $S^{3}$ complements of a two-component link which is a connected sum of a knot $\mathcal{K}$ and the Hopf link. For this class of links, the Chern-Simons theory provides the necessary setting to visualise the $m$-moment of the reduced density matrix as a three-manifold invariant $Z\left(M_{\mathcal{K}_{m}}\right)$, which is the partition function of $M_{\mathcal{K}_{m}}$. Here $M_{\mathcal{K}_{m}}$ is a closed 3-manifold associated with the knot $\mathcal{K}_{m}$, where $\mathcal{K}_{m}$ is a connected sum of $m$-copies of $\mathcal{K}$ (i.e., $\mathcal{K} \# \mathcal{K} \ldots \# \mathcal{K}$ ) which mimics the well-known replica method. We analayse the partition functions $Z\left(M_{\mathcal{K}_{m}}\right)$ for $\mathrm{SU}(2)$ and $\mathrm{SO}(3)$ gauge groups, in the limit of the large Chern-Simons coupling $k$. For $\mathrm{SU}(2)$ group, we show that $Z\left(M_{\mathcal{K}_{m}}\right)$ can grow at most polynomially in $k$. On the contrary, we conjecture that $Z\left(M_{\mathcal{K}_{m}}\right)$ for $\mathrm{SO}(3)$ group shows an exponential growth in $k$, where the leading term of $\ln Z\left(M_{\mathcal{K}_{m}}\right)$ is the hyperbolic volume of the knot complement $S^{3} \backslash \mathcal{K}_{m}$. We further propose that the Rényi entropies associated with $\mathrm{SO}(3)$ group converge to a finite value in the large $k$ limit. We present some examples to validate our conjecture and proposal.

Keywords: Chern-Simons Theories, Conformal Field Theory, Topological Field Theories, Wilson, 't Hooft and Polyakov loops

ArXiv EPRINT: 2106.03396 


\section{Contents}

1 Introduction 1

2 Preliminaries 3

2.1 Chern-Simons theory and multi-boundary states 3

2.2 Reduced density matrix and Rényi entropy 5

3 Bi-partite state: connected sum of a knot $\mathcal{K}$ and Hopf link 6

3.1 Reduced density matrices 6

$\begin{array}{lll}3.2 & \text { Rényi entropies and three-manifold invariants } & 8\end{array}$

$3.3 \mathrm{SU}(2)$ gauge group \& $m$-moments of density matrix 9

3.3.1 Large $k$ asymptotics: the polynomial growth 10

$\begin{array}{lll}3.4 & \mathrm{SO}(3) \text { gauge group \& } m \text {-moments of density matrix } & 10\end{array}$

3.4.1 Large $k$ asymptotics: the exponential growth 12

$\begin{array}{lll}3.4 .2 & \text { Large } k \text { limits of Rényi entropies } & 13\end{array}$

4 Numerical results for $\mathrm{SO}(3)$ group $\quad 14$

$\begin{array}{lll}4.1 & \text { Torus knots } & 15\end{array}$

$\begin{array}{lll}\text { 4.1.1 Trefoil knot: } 3 & 15\end{array}$

$\begin{array}{ll}\text { 4.1.2 Solomon's Seal knot: } 5_{1} & 17\end{array}$

$\begin{array}{lll}4.2 & \text { Non-torus knots } & 18\end{array}$

$\begin{array}{lll}4.2 .1 & \text { Figure-eight knot: } 4_{1} & 18\end{array}$

$\begin{array}{lll}\text { 4.2.2 Three-twist knot: } 5_{2} & 19\end{array}$

4.2.3 Stevedore knot: $6_{1} 22$

$\begin{array}{lll}4.2 .4 & 6_{2} \text { knot } & 22\end{array}$

$\begin{array}{lll}4.2 .5 & 6_{3} \text { knot } & 26\end{array}$

5 Conclusion $\quad 26$

A SO(3) Chern-Simons theory and hyperbolic volumes of $S^{3} \backslash \mathcal{K} \quad 29$

\section{Introduction}

The study of quantum entanglement and finding the possible patterns of entanglement that can emerge in a quantum field theory (QFT) is generally an important question in quantum mechanics and quantum information theory. However, due to large degrees of freedom, it is a difficult exercise to analyse the entanglement structures in a generic QFT. Nevertheless, a class of QFTs known as 'topological quantum field theories' (TQFT's) provides a tractable system to analyse entanglement structures. 


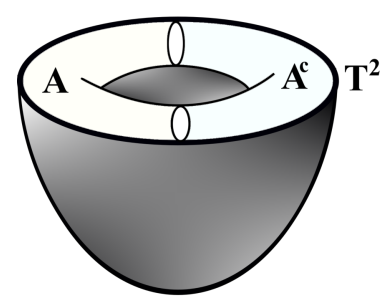

(a)

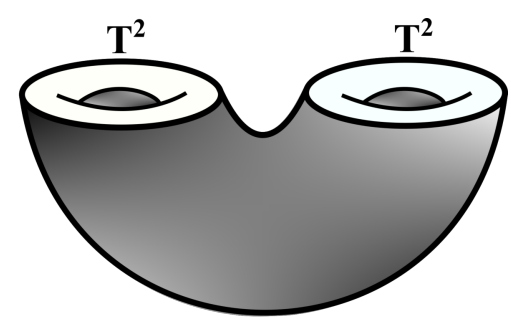

(b)

Figure 1. The two approaches to study the topological entanglement: the manifold in $(a)$ has a single torus boundary, which is bi-partitioned into spatially connected regions $A$ and $A^{c}$. The manifold in (b) has two disjoint torus boundaries.

The three-dimensional Chern-Simons theory is one such TQFT [1] which provides a natural framework to study invariants of knots, links, and three-manifolds. Interestingly, the Chern-Simons path integral on a three-manifold $M$ with boundary $\partial M=\Sigma$ is given by a state belonging to a finite-dimensional Hilbert space $\mathcal{H}_{\Sigma}$. Such a finite-dimensional Hilbert space is a key behind explicitly computing the entanglement measures.

There are two different topological set-ups to obtain the entanglement entropy in Chern-Simons theory as shown in figure 1. In figure 1(a), the manifold has a single torus boundary which is bi-partitioned into spatially connected regions $A$ and its complement $A^{c}$. When we trace out the region $A^{c}$, we obtain the reduced density matrix $\rho_{A}$ and the corresponding 'topological entanglement entropy'. Such an entropy is independent of the length or the area of the region $A$ or $A^{c}$ and have been studied in [2-4]. Another approach to study the topological entanglement was given in [5], where the three-manifold is actually a link complement ${ }^{1}$ of $S^{3}$ with two or more disjoint torus boundaries as shown in figure $1(b)$. The topological entanglement structure can be obtained by tracing out one of the boundary components, which is often termed as 'multi-boundary entanglement'. We refer the interested readers to [5-15] for the recent developments in this study. For the three-manifold $M$ whose boundary consists of multiple disjoint torus boundaries $\left(\partial M=\Sigma_{1} \sqcup \Sigma_{2} \sqcup \ldots \sqcup \Sigma_{n}\right)$, the associated Hilbert space is

$$
\mathcal{H}_{\partial M}=\mathcal{H}_{\Sigma_{1}} \otimes \mathcal{H}_{\Sigma_{2}} \otimes \ldots \otimes \mathcal{H}_{\Sigma_{n}}
$$

When $M$ is a link complement $\left(M=S^{3} \backslash \mathcal{L}\right)$, the probability amplitudes of the associated state $|\mathcal{L}\rangle$ are the Chern-Simons partition functions $Z\left(S^{3} ; \mathcal{L}\right)$ of $S^{3}$ in the presence of the link $\mathcal{L}$ (see [5]), which are proportional to the link invariants [1]. Therefore, the entanglement measures for such a state can be written in terms of the link invariants of $\mathcal{L}$.

In this work, we analyse the semiclassical (large $k$ ) asymptotics of the trace of the unnormalised reduced density matrices $(\operatorname{Tr}[\sigma(\mathcal{L})])$ of two-party states associated with the link complement $S^{3} \backslash \mathcal{L}$ for a class of two-component links $\mathcal{L}$ viewed as connected sum of prime knots $\mathcal{K}$ with the Hopf link $T_{2,2}$ :

$$
\mathcal{L}=\mathcal{K} \# T_{2,2} .
$$

\footnotetext{
${ }^{1}$ Given a link $\mathcal{L}$ embedded in $S^{3}$, the link complement $S^{3} \backslash \mathcal{L}$ is a three-dimensional manifold which is obtained by removing a tubular neighborhood around $\mathcal{L}$ from $S^{3}$, i.e $S^{3} \backslash \mathcal{L} \equiv S^{3}-\operatorname{interior}\left(\mathcal{L}_{\text {tub }}\right)$.
} 
Qualitatively, $\operatorname{Tr}\left[\sigma\left(\mathcal{K} \# T_{2,2}\right)\right]$ describes the Chern-Simons partition function of some closed three-manifold $M_{\mathcal{K}}$ (although the topology of $M_{\mathcal{K}}$ is not known to us) [9]. For the class of links (1.2), this trace is given as [5]:

$$
\operatorname{Tr}\left[\sigma\left(\mathcal{K} \# T_{2,2}\right)\right]=Z\left(M_{\mathcal{K}}\right)=\sum_{R}\left|H_{R}\left(\mathcal{K} ; q=e^{\frac{2 \pi i}{k+y}}\right)\right|^{2}
$$

where $H_{R}(\mathcal{K} ; q)$ are the reduced quantum invariants of knot $\mathcal{K}$ with $y$ being the dual Coxeter number of the gauge group (see the details in section 3). Such a trace (1.3) for $\mathrm{SU}(2)$ gauge group almost resembles the Turaev-Viro invariant $\operatorname{TV}_{r}\left(S^{3} \backslash \mathcal{K} ; t=e^{\frac{\pi i}{r}}\right)$ for the knot complement $S^{3} \backslash \mathcal{K}$ with $t^{2}=q$ and $r=(k+2)$ [16, 17]. In fact, this resemblance to $\mathrm{TV}_{r}\left(S^{3} \backslash \mathcal{K} ; t\right)$ motivates us to investigate the large $k$ asymptotics of (1.3) for the class of links $\mathcal{K} \# T_{2,2}$ within the Chern-Simons theory.

For $\mathrm{SU}(2)$ gauge group, we observe the trace (1.3) follows the same polynomial growth in $k$ as that of Turaev-Viro invariant $\operatorname{TV}_{r}\left(S^{3} \backslash \mathcal{K} ; t=e^{\frac{\pi i}{r}}\right)$ with $r=(k+2)$. Incidentally, the large $k$ asymptotics of Turaev-Viro invariant [17] evaluated at a different root of unity $t=e^{\frac{2 \pi i}{r^{\prime}}}$, with $r^{\prime}=(k+1)$ being an odd integer, captures the hyperbolic volume of the knot complement $S^{3} \backslash \mathcal{K}$. Such a change of variable naturally happens when we study the knot invariants in $\mathrm{SO}(3)$ Chern-Simons theory (we refer to section 3 for more details).

Hence our focus in this work is to investigate the trace (1.3) for the links of type $\mathcal{K} \# T_{2,2}$ within the context of $\mathrm{SO}(3)$ Chern-Simons theory. Interestingly, our numerical analysis, combined with the Kashaev's conjecture [18], is instrumental in extracting the geometrical features of $S^{3} \backslash \mathcal{K}$ from (1.3). Specifically, we conjecture an exponential growth of $\operatorname{Tr}\left[\sigma\left(\mathcal{K} \# T_{2,2}\right)\right]=Z\left(M_{\mathcal{K}}\right)$ with $k$, where the growth rate is determined by the hyperbolic volume of $S^{3} \backslash \mathcal{K}$.

We further propose that the Rényi entropies for $\mathrm{SO}(3)$ gauge group associated with the state $\left|\mathcal{K} \# T_{2,2}\right\rangle$ converge to a finite value as $k \rightarrow \infty$, although currently we do not have a geometric or topological interpretation of this limit.

The paper is organized as follows. In section 2, we discuss the preliminaries, including a brief discussion of our set-up. In section 3, we begin by analysing the reduced density matrices and their traces associated with the links of type $\mathcal{K} \# T_{2,2}$. We further analyse their large $k$ asymptotics for two gauge groups: $\mathrm{SU}(2)$ and $\mathrm{SO}(3)$, respectively. In section 4 , we present several examples where we do explicit numerical computations to verify the results conjectured and proposed in section 3 for the $\mathrm{SO}(3)$ group. We finally conclude in section 5 .

\section{Preliminaries}

\subsection{Chern-Simons theory and multi-boundary states}

Three-dimensional Chern-Simons theory based on a compact gauge group $G$ and coupling $k \in \mathbb{Z}$ is described by the following metric independent action:

$$
S(A)=\frac{k}{4 \pi} \int_{M} \operatorname{Tr}\left(A \wedge d A+\frac{2}{3} A \wedge A \wedge A\right) .
$$


Here $A=A_{\mu} d x^{\mu}$ is a matrix-valued gauge field, and $M$ denotes a three-manifold. The gauge-invariant operators are the Wilson loops. Given a knot $\mathcal{K}$ embedded in $M$, the Wilson loop operator is defined by taking the trace of the holonomy of $A$ around $\mathcal{K}$ :

$$
W_{R}(\mathcal{K})=\operatorname{Tr}_{R} P \exp \left(i \oint_{\mathcal{K}} A\right)
$$

Note that the knot $\mathcal{K}$ carries a representation $R$ of the gauge group $G$, and the trace is over the matrix corresponding to the representation $R$. Chern-Simons partition function, which encodes the topological information of the three-manifold $M$, is given by

$$
Z(M)=\int e^{i S(A)} \mathcal{D} A
$$

where $\mathcal{D} A$ denotes integration over all the gauge-invariant classes of connections. In fact, $Z(M)$ is referred to as a three-manifold invariant (up to an overall normalisation) in the literature.

The partition function of $M$ in the presence of knots and links is obtained by inserting the appropriate Wilson loop operators in the integral. For example, consider a link $\mathcal{L}$ made of component knots $\mathcal{K}_{1}, \mathcal{K}_{2}$ given by Wilson link operator $W_{R 1, R_{2}}(\mathcal{L})=W_{R_{1}}\left(\mathcal{K}_{1}\right) W_{R_{2}}\left(\mathcal{K}_{2}\right)$. Note that the representations $R_{1}, R_{2}$ are placed on the component knots $\mathcal{K}_{1}, \mathcal{K}_{2}$ respectively. The partition function in the presence of such a link is given by

$$
Z\left(M ; \mathcal{L}\left[R_{1}, R_{2}\right]\right)=\int e^{i S(A)} W_{R_{1}}\left(\mathcal{K}_{1}\right) W_{R_{2}}\left(\mathcal{K}_{2}\right) \mathcal{D} A
$$

Suppose the manifold is closed with no boundary, the link invariants $V_{R_{1}, R_{2}}(\mathcal{L})$ with the two components carrying representation $R_{1}, R_{2}$ are given by the expectation value of the Wilson link operator:

$$
V_{R_{1}, R_{2}}(\mathcal{L})=\frac{1}{Z(M)} \int e^{i S(A)} W_{R_{1}}\left(\mathcal{K}_{1}\right) W_{R_{2}}\left(\mathcal{K}_{2}\right) \mathcal{D} A
$$

When $M$ has a boundary $\Sigma$, the path integral of the theory on $M$ with the Wilson link $\mathcal{L}$ insertion is interpreted as a state $|\Psi\rangle \equiv|\mathcal{L}\rangle \in \mathcal{H}_{\Sigma}$. Further note that if we reverse the orientation of the boundary, the associated Hilbert space becomes the dual of the original Hilbert space:

$$
\mathcal{H}_{\Sigma^{*}}=\mathcal{H}_{\Sigma}^{*}
$$

As a result, there exists a natural pairing, the inner product $\langle\Phi \mid \Psi\rangle$ for any two states $|\Psi\rangle \in \mathcal{H}_{\Sigma}$ and $\langle\Phi| \in \mathcal{H}_{\Sigma^{*}}$. This technique can be used to compute the partition functions $Z(M)$ of complicated closed manifolds by gluing two disconnected pieces along their oppositely oriented boundary as shown in the figure 2 . When the boundary of the manifold $M$ consists of disjoint components, i.e., $\Sigma=\Sigma_{1} \sqcup \Sigma_{2}$, the Hilbert space associated with $\Sigma$ is the tensor product of Hilbert spaces associated with each component, i.e.

$$
\mathcal{H}_{\Sigma}=\mathcal{H}_{\Sigma_{1}} \otimes \mathcal{H}_{\Sigma_{2}}
$$

For the states $|\Psi\rangle \in \mathcal{H}_{\Sigma}$, we can study the entanglement structure by tracing out the Hilbert space $\mathcal{H}_{\Sigma_{1}}$ or $\mathcal{H}_{\Sigma_{2}}$. In this work, we will consider the link complement manifold

$$
M=S^{3} \backslash \mathcal{L},
$$




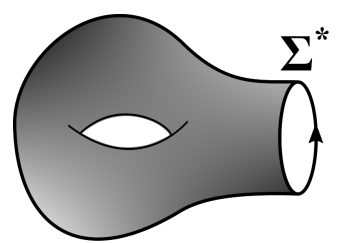

$\langle\phi|$

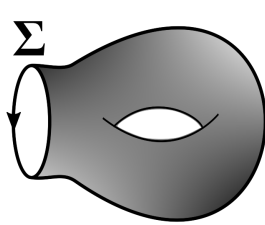

$|\psi\rangle$

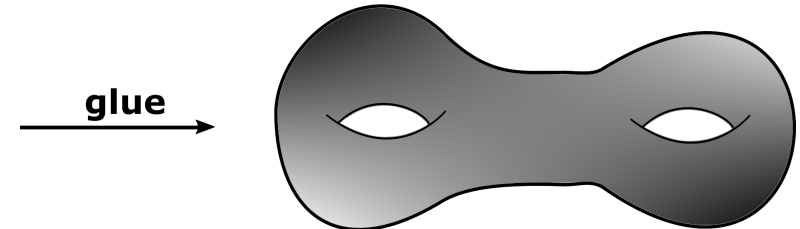

$\langle\phi \mid \psi\rangle$

Figure 2. Two manifolds on left with same boundary but opposite orientation. The path integral on these manifolds gives states $\langle\phi| \in \mathcal{H}_{\Sigma^{*}}$ and $|\psi\rangle \in \mathcal{H}_{\Sigma}$. The inner product $\langle\phi \mid \psi\rangle$ will be the partition function of a closed three-manifold (shown in right) obtained by gluing.

whose boundary is $\Sigma=T^{2} \sqcup T^{2}$ for any two-component link $\mathcal{L}$. We denote the state associated with $S^{3} \backslash \mathcal{L}$ as $|\mathcal{L}\rangle \in \mathcal{H}_{T^{2}} \times \mathcal{H}_{T^{2}}$. We will now review the essential steps [5] of computing such states. As discussed in ref. [1], the basis states of the Hilbert space $\mathcal{H}_{T^{2}}$ are in one-to-one correspondence with the integrable representations of the affine Lie algebra $\hat{\mathfrak{g}}_{k}$ at level $k$. Here $\mathfrak{g}$ represents the Lie algebra associated with the group $G$. The Chern-Simons path integral on a solid torus with a Wilson loop along the non-contractible cycle carrying integrable representation $\alpha$ is given by the basis state $\left|e_{\alpha}\right\rangle$ :

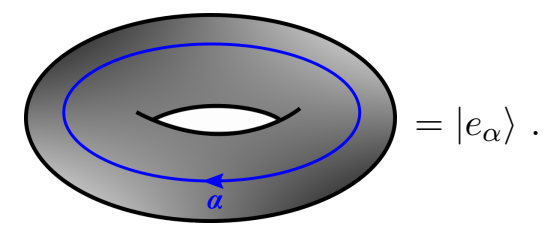

Using such basis states, we can expand the bipartite state $|\mathcal{L}\rangle$ as:

$$
|\mathcal{L}\rangle=\sum_{\alpha} \sum_{\beta} C_{\alpha \beta}\left|e_{\alpha}\right\rangle \otimes\left|e_{\beta}\right\rangle=\sum_{\alpha} \sum_{\beta} C_{\alpha \beta}\left|e_{\alpha}, e_{\beta}\right\rangle
$$

where $C_{\alpha \beta}$ are the complex coefficients given by partition function (2.4) [5]:

$$
C_{\alpha \beta}=\left\langle e_{\alpha}, e_{\beta} \mid \mathcal{L}\right\rangle=Z\left(S^{3} ; \mathcal{L}[\alpha, \beta]\right) .
$$

Hence the two-component link state can be written as

$$
|\mathcal{L}\rangle=\sum_{\alpha} \sum_{\beta} Z\left(S^{3} ; \mathcal{L}[\alpha, \beta]\right)\left|e_{\alpha}, e_{\beta}\right\rangle
$$

To study its entanglement properties, we require a reduced density matrix by partial tracing one of the two Hilbert spaces. We will now present these entanglement features in the following subsection.

\subsection{Reduced density matrix and Rényi entropy}

Given the link state $|\mathcal{L}\rangle \in \mathcal{H}_{1} \otimes \mathcal{H}_{2}$ in (2.12), the corresponding density matrix operator is

$$
\hat{\rho}(\mathcal{L})=|\mathcal{L}\rangle\langle\mathcal{L}| .
$$


The reduced density matrix is computed by tracing out one of the Hilbert space, say $\mathcal{H}_{2}$ :

$$
\rho(\mathcal{L})=\frac{\operatorname{Tr}_{\mathcal{H}_{2}}[\hat{\rho}(\mathcal{L})]}{\langle\mathcal{L} \mid \mathcal{L}\rangle}=\frac{1}{\langle\mathcal{L} \mid \mathcal{L}\rangle} \sum_{\alpha}\left\langle e_{\alpha}|\hat{\rho}(\mathcal{L})| e_{\alpha}\right\rangle
$$

where the factor $\langle\mathcal{L} \mid \mathcal{L}\rangle$ in the denominator ensures that the trace of $\rho(\mathcal{L})$ is unity. For convenience, we will work with the unnormalised version of the reduced density matrix as follows:

$$
\sigma(\mathcal{L})=\operatorname{Tr}_{\mathcal{H}_{2}}[\hat{\rho}(\mathcal{L})]
$$

The entanglement measures associated with $|\mathcal{L}\rangle$ can be computed from the matrix $\sigma(\mathcal{L})$. For example, the Rényi entropy of index $m$ can be written in terms of $m$-moments or the trace of the $m^{\text {th }}$ power of $\sigma(\mathcal{L})$ as following:

$$
\mathcal{R}_{m}=\frac{1}{1-m} \ln \left(\frac{\operatorname{Tr}\left[\sigma^{m}(\mathcal{L})\right]}{(\operatorname{Tr}[\sigma(\mathcal{L})])^{m}}\right) .
$$

The entanglement entropy is given as,

$$
\mathcal{E}=\lim _{m \rightarrow 1} \mathcal{R}_{m} .
$$

There also exists a minimum entropy which is controlled by the maximum eigenvalue of the reduced density matrix. Further, in the large $m$ limit, the corresponding Rényi entropy is minimum:

$$
\mathcal{R}_{\min } \equiv \mathcal{R}_{\infty}=\lim _{m \rightarrow \infty} \mathcal{R}_{m}
$$

In the next section, we will study some of the properties of the density matrices and the entanglement measures associated with a class of two-component links $\mathcal{L}$ which is a connected sum of a prime knot $\mathcal{K}$ with Hopf link.

\section{Bi-partite state: connected sum of a knot $\mathcal{K}$ and Hopf link}

In this work, we consider the two-component link $\mathcal{L}=\mathcal{K} \# T_{2,2}$ which is a connected sum of a prime knot $\mathcal{K}$ and the Hopf link $T_{2,2}$. A typical example of such a link is shown in the figure 3 when $\mathcal{K}$ is the figure-eight knot. In this section, we will closely analyse the bi-partite state $\left|\mathcal{K} \# T_{2,2}\right\rangle$ and its properties. In particular, our focus will be on the traces of the unnormalised reduced density matrices. As mentioned in the introduction, we will see that these traces have a close resemblance to the Turaev-Viro invariant [16, 17], motivating us to focus on the Chern-Simons theory based on $\mathrm{SU}(2)$ as well as $\mathrm{SO}(3)$ gauge groups.

We will first start with a generic discussion on the density matrices associated with $\left|\mathcal{K} \# T_{2,2}\right\rangle$ in the following subsection.

\subsection{Reduced density matrices}

Following the prescription given in the previous section, we can write the state associated with the link $\mathcal{K} \# T_{2,2}$ as

$$
\left|\mathcal{K} \# T_{2,2}\right\rangle=\sum_{\alpha, \beta} Z\left(S^{3}, \mathcal{K}_{\alpha} \# T_{2,2}[\alpha, \beta]\right)\left|e_{\alpha}, e_{\beta}\right\rangle,
$$




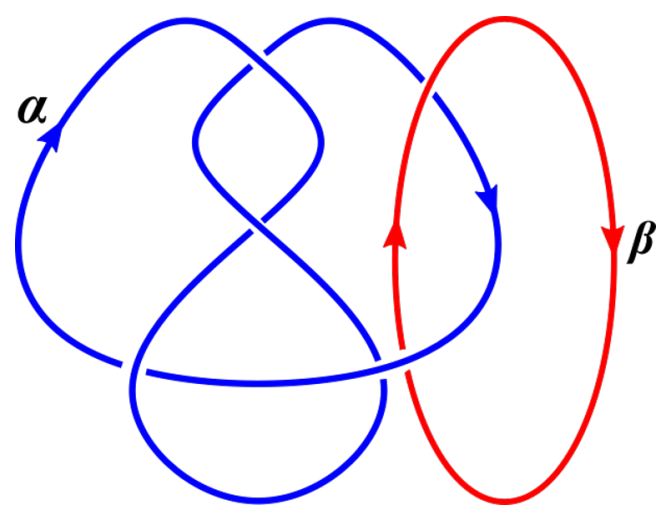

Figure 3. The two component link $4_{1} \# T_{2,2}$ which is the connected sum of figure-eight knot and Hopf link.

where the probability amplitudes are the Chern-Simons partition functions of $S^{3}$ in presence of the link $\mathcal{K} \# T_{2,2}$. The labels $\alpha$ and $\beta$ are the representations carried by $\mathcal{K}$ and unknot components of the link respectively (as shown in the example in figure 3 ). This partition function can be computed following ref. [1] and is given as,

$$
Z\left(S^{3}, \mathcal{K}_{\alpha} \# T_{2,2}[\alpha, \beta]\right)=\frac{Z\left(S^{3}, \mathcal{K}_{\alpha}\right) Z\left(S^{3}, T_{2,2}[\alpha, \beta]\right)}{Z\left(S^{3}, U_{\alpha}\right)}=Z\left(S^{3}, \mathcal{K}_{\alpha}\right) \frac{\mathcal{S}_{\alpha \beta}}{\mathcal{S}_{0 \alpha}},
$$

where $U_{\alpha}$ is the unknot with representation $\alpha$. In the last equality, the partition functions of Hopf link and unknot are given in terms of the elements of the modular transformation matrix $^{2} \mathcal{S}$. Thus we can write the state as:

$$
\left|\mathcal{K} \# T_{2,2}\right\rangle=\sum_{\alpha, \beta} Z\left(S^{3}, \mathcal{K}_{\alpha}\right) \frac{\mathcal{S}_{\alpha \beta}}{\mathcal{S}_{0 \alpha}}\left|e_{\alpha}, e_{\beta}\right\rangle
$$

As the entanglement properties of a state do not change under a local unitary change of the basis, we simplify the above state by performing the following transformation:

$$
\left|e_{\beta}\right\rangle=\sum_{x} \mathcal{S}_{\beta x}^{*}\left|e_{x}\right\rangle
$$

Substituting such a transformation in (3.3) and using the unitary property of the $\mathcal{S}$ matrix, we get

$$
\left|\mathcal{K} \# T_{2,2}\right\rangle=\sum_{\alpha} \frac{Z\left(S^{3}, \mathcal{K}_{\alpha}\right)}{\mathcal{S}_{0 \alpha}}\left|e_{\alpha}, e_{\alpha}\right\rangle
$$

Interestingly, the probability amplitudes appearing in the above state are precisely the reduced quantum invariants ${ }^{3}$ of the knot $\mathcal{K}$ evaluated at a specific root of unity [1]:

$$
\frac{Z\left(S^{3}, \mathcal{K}_{\alpha}\right)}{\mathcal{S}_{0 \alpha}}=\frac{Z\left(S^{3}, \mathcal{K}_{\alpha}\right) / Z\left(S^{3}\right)}{\mathcal{S}_{0 \alpha} / Z\left(S^{3}\right)}=\frac{V_{\alpha}(\mathcal{K})}{V_{\alpha}(U)}=H_{\alpha}\left(\mathcal{K} ; q=e^{\frac{2 \pi i}{k+y}}\right)
$$

\footnotetext{
${ }^{2}$ The operator $\mathcal{S}$ is one of the generators of the modular group $\operatorname{SL}(2, \mathbb{Z})$. In the above discussion, the operator $\mathcal{S}$ is written in a matrix form with element $\mathcal{S}_{\alpha \beta}$, where $\alpha$ and $\beta$ label the integrable representations of affine Lie algebra $\mathfrak{g}_{k}$ at level $k$. For $\mathfrak{s u}(2)_{k}$ and $\mathfrak{s o}(3)_{k}$ algebras, the matrix $\mathcal{S}$ is symmetric, unitary and satisfies $\mathcal{S}^{*}=\mathcal{S}$.

${ }^{3}$ The reduced invariants are normalised such that the unknot invariant is unity, i.e. $H_{\alpha}(U ; q)=1$.
} 
where $H_{\alpha}(\mathcal{K} ; q)$ are the reduced invariants of knot $\mathcal{K}$ evaluated at $q=e^{\frac{2 \pi i}{k+y}}$ with $y$ being the dual Coxeter number of the gauge group. Thus we arrive at the following state:

$$
\left|\mathcal{K} \# T_{2,2}\right\rangle=\sum_{\alpha} H_{\alpha}\left(\mathcal{K} ; q=e^{\frac{2 \pi i}{k+y}}\right)\left|e_{\alpha}, e_{\alpha}\right\rangle .
$$

The unnormalised reduced density matrix (2.15) for this state is a diagonal matrix:

$$
\sigma\left(\mathcal{K} \# T_{2,2}\right)=\operatorname{diag}\left\{\left|H_{\alpha}\left(\mathcal{K} ; q=e^{\frac{2 \pi i}{k+y}}\right)\right|^{2} ; \quad \alpha \in \text { Integrable representations }\right\},
$$

where the entries are labeled by various integrable representations $\alpha$. The $m$-moment or the trace of the $m^{\text {th }}$ power of this matrix is:

$$
\operatorname{Tr}\left[\sigma^{m}\left(\mathcal{K} \# T_{2,2}\right)\right]=\sum_{\alpha}\left|H_{\alpha}\left(\mathcal{K} ; q=e^{\frac{2 \pi i}{k+y}}\right)\right|^{2 m} .
$$

We also know that the trace of the reduced density matrix $\sigma\left(\mathcal{K} \# T_{2,2}\right)$ can be viewed as gluing oppositely oriented torus boundaries resulting in a closed three-manifold without any boundary. Some examples have been qualitatively discussed in ref. [9]. For clarity, let us look at the simplest link $\mathcal{L}=U \# T_{2,2}$ involving the unknot $U$ whose Jones polynomial is 1 . Clearly,

$$
\operatorname{Tr}\left[\sigma\left(U \# T_{2,2}\right)\right]=\sum_{\alpha}(1)=\operatorname{dim} \mathcal{H}_{T^{2}}=Z\left(T^{2} \times S^{1}\right),
$$

giving the Chern-Simons partition function of $M_{U} \equiv T^{2} \times S^{1}$. Replacing $U$ by a non-trivial prime knot $\mathcal{K}$ would eventually give us:

$$
\operatorname{Tr}\left[\sigma\left(\mathcal{K} \# T_{2,2}\right)\right]=\sum_{\alpha}\left|H_{\alpha}\left(\mathcal{K} ; q=e^{\frac{2 \pi i}{k+y}}\right)\right|^{2}=Z\left(M_{\mathcal{K}}\right)
$$

for some closed three-manifold $M_{\mathcal{K}}$, though we cannot specify the explicit topology of $M_{\mathcal{K}}$. Further, the $m$-moment of the unnormalised reduced density matrix $\sigma\left(\mathcal{K} \# T_{2,2}\right)$ can be viewed as joining $m$-copies of the oppositely oriented $T^{2}$ boundaries. This is called the replica method [19] and it again results in a closed three-manifold. In the following subsection, we will present the knot theoretic interpretation of the replica method and their relations to $m$-moments of $\sigma\left(\mathcal{K} \# T_{2,2}\right)$ defining three-manifold invariants. We will show that the Rényi entropies of index $m$ can be written in terms of these three-manifold invariants.

\subsection{Rényi entropies and three-manifold invariants}

The Rényi entropy of index $m$ associated with the reduced density matrix of state $\left|\mathcal{K} \# T_{2,2}\right\rangle$ can be computed as:

$$
\mathcal{R}_{m}=\frac{1}{1-m} \ln \left[\frac{\operatorname{Tr}\left[\sigma^{m}\left(\mathcal{K} \# T_{2,2}\right)\right]}{\left(\operatorname{Tr}\left[\sigma\left(\mathcal{K} \# T_{2,2}\right)\right]\right)^{m}}\right]=\frac{1}{1-m} \ln \left[\frac{\operatorname{Tr}\left[\sigma^{m}\left(\mathcal{K} \# T_{2,2}\right)\right]}{Z\left(M_{\mathcal{K}}\right)^{m}}\right] .
$$

We aim to write the numerator of the logarithmic term also as a three-manifold invariant. Just like our discussion that $\operatorname{Tr}\left[\sigma\left(\mathcal{K} \# T_{2,2}\right)\right]=Z\left(M_{\mathcal{K}}\right)$, a similar topological attribution is 
also true for the higher moments of the reduced density matrix. We will now elaborate the knot theoretic picture of the replica trick to obtain $\operatorname{Tr}\left[\sigma^{m}\left(\mathcal{K} \# T_{2,2}\right)\right]$.

Replica method involving $m$-copies is achievable by replacing $\mathcal{K}$ by the following connected sum of knots:

$$
\mathcal{K}_{m} \equiv \underbrace{\mathcal{K} \# \mathcal{K} \# \ldots \# \mathcal{K}}_{m} .
$$

Incidentally, the reduced knot invariants for connected sum of two knots $\mathcal{K}_{1} \# \mathcal{K}_{2}$ is equal to the product of the reduced knot invariants for knots $\mathcal{K}_{1}$ and $\mathcal{K}_{2}$. Using this fact and (3.9), we will have:

$$
\operatorname{Tr}\left[\sigma\left(\mathcal{K}_{m} \# T_{2,2}\right)\right]=\sum_{\alpha}\left|H_{\alpha}\left(\mathcal{K}_{m} ; q\right)\right|^{2}=\sum_{\alpha}\left|H_{\alpha}(\mathcal{K} ; q)\right|^{2 m}=\operatorname{Tr}\left[\sigma^{m}\left(\mathcal{K} \# T_{2,2}\right)\right],
$$

with $q=e^{\frac{2 \pi i}{k+y}}$. Thus using (3.11) in (3.14), we realize:

$$
\operatorname{Tr}\left[\sigma^{m}\left(\mathcal{K} \# T_{2,2}\right)\right]=Z\left(M_{\mathcal{K}_{m}}\right)
$$

and hence we can write the Rényi entropies in terms of three-manifold invariants:

$$
\mathcal{R}_{m}=\frac{1}{1-m} \ln \left[\frac{Z\left(M_{\mathcal{K}_{m}}\right)}{Z\left(M_{\mathcal{K}}\right)^{m}}\right] \text {. }
$$

So far, our discussion has been for a generic gauge group. In the following subsections, we consider the cases when the gauge group is $\mathrm{SU}(2)$ and $\mathrm{SO}(3$, respectively. In particular, we focus on the large $k$ asymptotics of the traces $\operatorname{Tr}\left[\sigma^{m}\left(\mathcal{K} \# T_{2,2}\right)\right]=Z\left(M_{\mathcal{K}_{m}}\right)$, which almost resembles the Turaev-Viro invariants evaluated at certain roots of unity [17]. We start with the $\mathrm{SU}(2)$ case in the following subsection.

\section{3 $\mathrm{SU}(2)$ gauge group \& $m$-moments of density matrix}

The integrable representations for the affine algebra $\mathfrak{s u}(2)_{k}$ are given as:

$$
\{\underbrace{\square \prod \square}_{\alpha}: 0 \leq \alpha \leq k\} \text {. }
$$

Hence the basis states of $\mathcal{H}_{T^{2}}$ can be accordingly labeled:

$$
\operatorname{basis}\left(\mathcal{H}_{T^{2}}\right)=\{\left|e_{\alpha}\right\rangle: \alpha \equiv \underbrace{\square \amalg \Pi \rrbracket}_{\alpha}\} .
$$

Using (3.7) and noting that the reduced invariants $H_{\alpha}$ are the colored Jones polynomials, the state is written as:

$$
\left|\mathcal{K} \# T_{2,2}\right\rangle=\sum_{\alpha=0}^{k} J_{\alpha}\left(\mathcal{K} ; e^{\frac{2 \pi i}{k+2}}\right)\left|e_{\alpha}, e_{\alpha}\right\rangle
$$

and the $m$-moment or the trace of the $m^{\text {th }}$ power of the unnormalised reduced density matrix associated with $\left|\mathcal{K} \# T_{2,2}\right\rangle$ will be:

$$
\operatorname{Tr}\left[\sigma^{m}\left(\mathcal{K} \# T_{2,2}\right)\right]=\sum_{\alpha=0}^{k}\left|J_{\alpha}\left(\mathcal{K} ; e^{\frac{2 \pi i}{k+2}}\right)\right|^{2 m} .
$$

We will now show that the large $k$ asymptotics of (3.20) is the same as that of Turaev-Viro invariant [16] of $S^{3} \backslash \mathcal{K}_{m}$ at a specific root of unity. 


\subsubsection{Large $k$ asymptotics: the polynomial growth}

Following [17], the Turaev-Viro invariant of $S^{3} \backslash \mathcal{K}_{m}$ evaluated at $e^{\frac{\pi i}{k+2}}$ with integer $k \geq 1$ can be written in terms of values of its colored Jones polynomials: ${ }^{4}$

$$
\begin{aligned}
\operatorname{TV}_{k+2}\left(S^{3} \backslash \mathcal{K}_{m} ; e^{\frac{\pi i}{k+2}}\right) & =\frac{2}{k+2} \sum_{\alpha=0}^{k} \sin ^{2}\left(\frac{\pi \alpha+\pi}{k+2}\right)\left|J_{\alpha}\left(\mathcal{K}_{m} ; e^{\frac{2 \pi i}{k+2}}\right)\right|^{2} \\
& =\frac{2}{k+2} \sum_{\alpha=0}^{k} \sin ^{2}\left(\frac{\pi \alpha+\pi}{k+2}\right)\left|J_{\alpha}\left(\mathcal{K} ; e^{\frac{2 \pi i}{k+2}}\right)\right|^{2 m}
\end{aligned}
$$

We also have the following inequality which is valid for $0 \leq \alpha \leq k$ :

$$
\frac{1}{(k+2)^{2}} \leq \sin ^{2}\left(\frac{\pi \alpha+\pi}{k+2}\right) \leq 1
$$

Using (3.20) and (3.21), and applying (3.22), we obtain the following inequalities:

$$
\frac{2}{(k+2)^{3}} \operatorname{Tr}\left[\sigma^{m}\left(\mathcal{K} \# T_{2,2}\right)\right] \leq \operatorname{TV}_{k+2}\left(S^{3} \backslash \mathcal{K}_{m} ; e^{\frac{\pi i}{k+2}}\right) \leq \frac{2}{k+2} \operatorname{Tr}\left[\sigma^{m}\left(\mathcal{K} \# T_{2,2}\right)\right]
$$

Further, as mentioned in [17], the growth of $\operatorname{TV}_{r}\left(M ; e^{\frac{\pi i}{r}}\right)$ is expected to be a polynomial in $r$ for 3-manifolds $M$. Thus the above inequality tells that $\operatorname{Tr}\left[\sigma^{m}\left(\mathcal{K} \# T_{2,2}\right)\right]$ can grow at most polynomially in $k$. In particular, taking log, dividing by $k$ and letting $k \rightarrow \infty$ in the expression (3.23), we get:

$$
\lim _{k \rightarrow \infty} \frac{\ln \operatorname{Tr}\left[\sigma^{m}\left(\mathcal{K} \# T_{2,2}\right)\right]}{k}=\lim _{k \rightarrow \infty} \frac{\ln \operatorname{TV}_{k+2}\left(S^{3} \backslash \mathcal{K}_{m} ; e^{\frac{\pi i}{k+2}}\right)}{k}=0 .
$$

Since the trace $\operatorname{Tr}\left[\sigma^{m}\left(\mathcal{K} \# T_{2,2}\right)\right]$ in (3.20) can be viewed as the $\mathrm{SU}(2)$ partition function $Z\left(M_{\mathcal{K}_{m}}\right)$, we have the following limit:

$$
\lim _{k \rightarrow \infty} \frac{\ln Z\left(M_{\mathcal{K}_{m}}\right)}{k}=\lim _{k \rightarrow \infty} \frac{\ln \operatorname{TV}_{k+2}\left(S^{3} \backslash \mathcal{K}_{m} ; e^{\frac{\pi i}{k+2}}\right)}{k}=0 .
$$

In the following section, we investigate the large $k$ asymptotics for $\mathrm{SO}(3)$ gauge group.

\section{$3.4 \mathrm{SO}(3)$ gauge group \& $m$-moments of density matrix}

Here, we will confine to Chern-Simons theory based on $\mathrm{SO}(3)$ gauge group where the level $k$ is a positive even integer. Since the group $\mathrm{SO}(3)$ has rank one, the integrable representations are given by the Dynkin labels $\alpha$ of the highest weights in the following range:

$$
0 \leq \alpha \leq k ; \quad \alpha \in \mathbb{Z}, k \in 2 \mathbb{Z} .
$$

\footnotetext{
${ }^{4}$ The colored Jones $J_{\alpha}(\mathcal{K} ; q)$ here are the reduced invariants which are normalised such that $J_{\alpha}(U ; q)$ is unity as mentioned in footnote 3 . The invariants appearing in [17] follow a different normalisation where $J_{\alpha}(U ; q)$ is equal to the $q$-number $[\alpha+1]$. The Turaev-Viro invariant written in (3.21) takes care of this.
} 
Using the group theoretical fact that the representation of $\mathrm{SO}(3)$ with Dynkin label $\alpha$ is isomorphic to the representation of $\mathrm{SU}(2)$ with Dynkin label $2 \alpha$, we can write the list of integrable representations of $\mathfrak{s o}(3)_{k}$ in terms of following Young diagrams:

$$
\{\underbrace{\square \prod \square}_{2 \alpha}: 0 \leq \alpha \leq k\} \text {. }
$$

Hence the basis states of $\mathcal{H}_{T^{2}}$ can be accordingly labeled:

$$
\operatorname{basis}\left(\mathcal{H}_{T^{2}}\right)=\{\left|e_{\alpha}\right\rangle: \alpha \equiv \underbrace{\square \amalg \square}_{2 \alpha}\} .
$$

Thus, the state (3.7) associated with the link $\mathcal{K} \# T_{2,2}$ can be written as

$$
\left|\mathcal{K} \# T_{2,2}\right\rangle=\sum_{\alpha=0}^{k} W_{\alpha}\left(\mathcal{K} ; e^{\frac{2 \pi i}{k+1}}\right)\left|e_{\alpha}, e_{\alpha}\right\rangle
$$

where $W_{\alpha}(\mathcal{K} ; q)$ are polynomials in $q$ and are the colored knot invariants for $\mathrm{SO}(3)$ group. ${ }^{5}$ Using the isomorphism between the $\mathrm{SO}(3)$ and $\mathrm{SU}(2)$ homologies, we can convert these $\mathrm{SO}(3)$ invariants into the colored Jones invariants. The two invariants are related as (we refer to [20] for some explicit examples):

$$
W_{\alpha}(\mathcal{K} ; q)=J_{2 \alpha}\left(\mathcal{K} ; q^{2}\right)
$$

where $J_{2 \alpha}$ is the colored Jones invariant evaluated for the spin $\alpha$ representation of $\mathrm{SU}(2)$ group. Thus we can rewrite the state in terms of the colored Jones invariants as:

$$
\left|\mathcal{K} \# T_{2,2}\right\rangle=\sum_{\alpha=0}^{k} J_{2 \alpha}\left(\mathcal{K} ; e^{\frac{4 \pi i}{k+1}}\right)\left|e_{\alpha}, e_{\alpha}\right\rangle
$$

The $m$-moment or the trace of the $m^{\text {th }}$ power of the unnormalised reduced density matrix associated with $\left|\mathcal{K} \# T_{2,2}\right\rangle$ will be:

$$
\operatorname{Tr}\left[\sigma^{m}\left(\mathcal{K} \# T_{2,2}\right)\right]=\sum_{\alpha=0}^{k}\left|J_{2 \alpha}\left(\mathcal{K} ; e^{\frac{4 \pi i}{k+1}}\right)\right|^{2 m} .
$$

One of the key questions we plan to investigate is the large $k$ behaviour of these $m$ - moments. We believe that they must capture geometrical features of the three-manifolds. This will be the focus in the following subsection.

\footnotetext{
${ }^{5}$ The reduced Chern-Simons invariants of knots for $\mathrm{SO}(N)$ gauge group are called the colored Kauffman polynomials: $\operatorname{Kauff}_{\alpha}(\mathcal{K} ; a, q)$, where the two variables are $a=q^{N-1}$ and $q=e^{\frac{2 \pi i}{k+N-2}}$. For the group $\operatorname{SO}(3)$, we define $W_{\alpha}(\mathcal{K} ; q) \equiv \operatorname{Kauff}_{\alpha}\left(\mathcal{K} ; q^{2}, q\right)$ with $q=e^{\frac{2 \pi i}{k+1}}$ which enter as probability amplitudes in (3.29).
} 


\subsubsection{Large $k$ asymptotics: the exponential growth}

We numerically checked that the colored Jones $J_{2 \alpha}\left(\mathcal{K} ; e^{\frac{4 \pi i}{k+1}}\right)$ has a maximum value when the color is $2 \alpha=k$ (note that $k$ is a positive even integer). Even though this observation was validated for some knots, we do not have an analytic argument.

For the $\mathrm{SO}(3)$ Chern-Simons theory where coupling $k \in 2 \mathbb{Z}$ and $\sigma\left(\mathcal{K} \# T_{2,2}\right)$ is the unnormalised reduced density matrix associated with the state $\left|\mathcal{K} \# T_{2,2}\right\rangle(\mathcal{K}$ is a prime knot and $T_{2,2}$ is the Hopf link), we put forth the following conjecture.

Conjecture 1. The large $k$ asymptotics of the trace of the $m^{\text {th }}$ power of the matrix $\sigma\left(\mathcal{K} \# T_{2,2}\right)$ captures the hyperbolic volume of $S^{3} \backslash \mathcal{K}$ :

$$
\lim _{k \rightarrow \infty} \frac{\ln \operatorname{Tr}\left[\sigma^{m}\left(\mathcal{K} \# T_{2,2}\right)\right]}{k}=m \frac{\operatorname{Vol}\left(S^{3} \backslash \mathcal{K}\right)}{2 \pi} .
$$

Proof. Using our numerical result that the colored Jones $J_{k}\left(\mathcal{K} ; e^{\frac{4 \pi i}{k+1}}\right)$ has maximum value, we can write the following inequality for (3.32):

$$
\left|J_{k}\left(\mathcal{K} ; e^{\frac{4 \pi i}{k+1}}\right)\right|^{2 m} \leq \operatorname{Tr}\left[\sigma^{m}\left(\mathcal{K} \# T_{2,2}\right)\right] \leq(k+1)\left|J_{k}\left(\mathcal{K} ; e^{\frac{4 \pi i}{k+1}}\right)\right|^{2 m} .
$$

Taking log, dividing by $k$ and then taking $k \rightarrow \infty$ limit will give

$$
\lim _{k \rightarrow \infty} \frac{\ln \operatorname{Tr}\left[\sigma^{m}\left(\mathcal{K} \# T_{2,2}\right)\right]}{k}=\lim _{k \rightarrow \infty} \frac{\ln \left|J_{k}\left(\mathcal{K} ; e^{\frac{4 \pi i}{k+1}}\right)\right|^{2 m}}{k} .
$$

To write the r.h.s. of the above equation as volume, we will use the following relation between the colored Jones invariant and the Kashaev's invariant [21]:

$$
\left|\operatorname{Kas}_{n}(\mathcal{K} ; q)\right|=\left|J_{n-1}(\mathcal{K} ; q)\right|, \quad q=\exp \left(\frac{2 \pi i a}{n}\right), \quad \operatorname{gcd}(a, n)=1,
$$

where $\operatorname{Kas}_{n}(\mathcal{K} ; q)$ is the Kashaev's invariant of the knot $\mathcal{K}$ and the variable $q$ is taken to be a primitive $n^{\text {th }}$ root of unity. In the large $n$ limit, the above Kashaev's invariant grows exponentially obeying the following conjecture [18]:

$$
\lim _{n \rightarrow \infty} \frac{\ln \left|\operatorname{Kas}_{n}\left(\mathcal{K} ; e^{\frac{2 \pi i a}{n}}\right)\right|}{n}=\frac{\operatorname{Vol}\left(S^{3} \backslash \mathcal{K}\right)}{2 \pi a} .
$$

That is, its growth rate is determined by the hyperbolic volume of $S^{3} \backslash \mathcal{K}$. This conjecture has been verified for $a=1$ case in refs. [18, 22] for many hyperbolic knots. For the $\mathrm{SO}(3)$ invariants we are studying, we have $n=(k+1)$, which is odd. Hence $a=2$ is also allowed in (3.36) and (3.37). We have checked the $a=2$ case of (3.37) in appendix A for some torus and non-torus knots. From the above discussion, we can now state that the $m$-moment of the matrix $\sigma$ for the class of two-component links $\mathcal{K} \# T_{2,2}$ must obey the following in the large $k$ limit:

$$
\lim _{k \rightarrow \infty} \frac{\ln \operatorname{Tr}\left[\sigma^{m}\left(\mathcal{K} \# T_{2,2}\right)\right]}{k}=\lim _{k \rightarrow \infty} \frac{\ln \left|J_{k}\left(\mathcal{K} ; e^{\frac{4 \pi i}{k+1}}\right)\right|^{2 m}}{k}=2 m \frac{\operatorname{Vol}\left(S^{3} \backslash \mathcal{K}\right)}{4 \pi},
$$

which gives our conjectured result (3.33). 
Further, following our earlier arguments that $\operatorname{Tr}\left[\sigma\left(\mathcal{K} \# T_{2,2}\right)\right]$ is the partition function $Z\left(M_{\mathcal{K}}\right)$, the conjecture $(3.33)$ leads to the following corollary:

Corollary 1. For any prime knot $\mathcal{K}$, there exists a closed three-manifold $M_{\mathcal{K}}$ such that the large $k$ asymptotics of the corresponding $S O(3)$ Chern-Simons partition function $Z\left(M_{\mathcal{K}}\right)$ determines the hyperbolic volume of $S^{3} \backslash \mathcal{K}$ :

$$
\lim _{k \rightarrow \infty} \frac{\ln Z\left(M_{\mathcal{K}}\right)}{k}=\frac{\operatorname{Vol}\left(S^{3} \backslash \mathcal{K}\right)}{2 \pi} .
$$

Thus, even though the explicit topology of $M_{\mathcal{K}}$ is not visualisable, the leading large $k$ behaviour of the $\mathrm{SO}(3)$ Chern-Simons partition function $Z\left(M_{\mathcal{K}}\right)$ captures the hyperbolic volume of $S^{3} \backslash \mathcal{K}$.

Moreover, as we have shown earlier in (3.16) that the Rényi entropies can be written in terms of the partition functions $Z\left(M_{\mathcal{K}_{m}}\right)$, it will be important to study the large $k$ asymptotics of the Rényi entropies. This will be the content of the following subsection.

\subsubsection{Large $k$ limits of Rényi entropies}

We have already discussed that the large $k$ asymptotics of $\operatorname{Tr}\left[\sigma^{m}\left(\mathcal{K} \# T_{2,2}\right)\right]$ or $Z\left(M_{\mathcal{K}_{m}}\right)$ is governed by the asymptotics of $\left|J_{k}\left(\mathcal{K} ; e^{\frac{4 \pi i}{k+1}}\right)\right|$. Since $\left|J_{k}\left(\mathcal{K} ; e^{\frac{4 \pi i}{k+1}}\right)\right|=\left|\operatorname{Kas}_{k+1}\left(\mathcal{K} ; e^{\frac{4 \pi i}{k+1}}\right)\right|$ from (3.36), it suffices to obtain the full asymptotics of $\left|\operatorname{Kas}_{k+1}\left(\mathcal{K} ; e^{\frac{4 \pi i}{k+1}}\right)\right|$. Setting $n=$ $(k+1)$, the following functional form for the Kashaev's invariant in the large $n$ limit was conjectured in [22]:

$$
\frac{2 \pi}{n} \ln \left|\operatorname{Kas}_{n}\left(\mathcal{K} ; q=e^{\frac{2 \pi i}{n}}\right)\right| \sim c_{1}(\mathcal{K})+c_{2}(\mathcal{K}) \frac{2 \pi}{n} \ln n+\frac{c_{3}(\mathcal{K})}{n}+\frac{c_{4}(\mathcal{K})}{n^{2}},
$$

where $c_{i}(\mathcal{K})$ are knot dependent constants such that [22]:

$$
c_{1}(\mathcal{K})=\operatorname{Vol}\left(S^{3} \backslash \mathcal{K}\right) ; \quad c_{2}(\mathcal{K})=\frac{3}{2} .
$$

Doing the numerical analysis for some of the non-trivial prime knots, we find that we can modify (3.40) for $q=e^{\frac{4 \pi i}{n}}$ as following:

$$
\frac{4 \pi}{n} \ln \left|\operatorname{Kas}_{n}\left(\mathcal{K} ; q=e^{\frac{4 \pi i}{n}}\right)\right| \sim a_{1}(\mathcal{K})+a_{2}(\mathcal{K}) \frac{4 \pi}{n} \ln n+\frac{a_{3}(\mathcal{K})}{n}+\frac{a_{4}(\mathcal{K})}{n^{2}},
$$

where $a_{i}(\mathcal{K})$ are constants such that:

$$
a_{1}(\mathcal{K})=\operatorname{Vol}\left(S^{3} \backslash \mathcal{K}\right) ; \quad a_{2}(\mathcal{K})=\frac{3}{2} .
$$

As a result, we will have the following asymptotics:

$$
\ln \left|J_{k}\left(\mathcal{K}, q=e^{\frac{4 \pi i}{k+1}}\right)\right| \sim \frac{\operatorname{Vol}\left(S^{3} \backslash \mathcal{K}\right)}{4 \pi} k+\ln k^{3 / 2}+\frac{a_{3}(\mathcal{K})}{4 \pi}+\frac{a_{4}(\mathcal{K})}{4 \pi k} .
$$

From (3.9) and (3.15), we will have:

$$
\frac{Z\left(M_{\mathcal{K}_{m}}\right)}{\left|J_{k}\left(\mathcal{K}, e^{\frac{4 \pi i}{k+1}}\right)\right|^{2 m}}=\sum_{\alpha=0}^{k}\left|\frac{J_{2 \alpha}\left(\mathcal{K} ; e^{\frac{4 \pi i}{k+1}}\right)}{J_{k}\left(\mathcal{K} ; e^{\frac{4 \pi i}{k+1}}\right)}\right|^{2 m} \equiv X_{m}(\mathcal{K} ; k) .
$$


Using (3.44), we have following asymptotics for large $k$ :

$$
Z\left(M_{\mathcal{K}_{m}}\right) \sim k^{3 m} \exp \left[\frac{m \operatorname{Vol}\left(S^{3} \backslash \mathcal{K}\right)}{2 \pi} k\right] \times\left(\exp \left[m \frac{a_{3}(\mathcal{K})}{2 \pi}+m \frac{a_{4}(\mathcal{K})}{2 \pi k}\right] X_{m}(\mathcal{K} ; k)\right) .
$$

From the numerical computations for several knots, we observe that the term in the parentheses is convergent as $k \rightarrow \infty$. So we define a knot dependent constant $C_{m}$ as:

$$
C_{m}(\mathcal{K})=\lim _{k \rightarrow \infty} \exp \left[m \frac{a_{3}(\mathcal{K})}{2 \pi}+m \frac{a_{4}(\mathcal{K})}{2 \pi k}\right] X_{m}(\mathcal{K} ; k) .
$$

Unfortunately, we do not have an analytical way to compute $C_{m}(\mathcal{K})$ and we restrict to the numerical computations. To compute $C_{m}(\mathcal{K})$, we define a function

$$
F_{m}(\mathcal{K}) \equiv Z\left(M_{\mathcal{K}_{m}}\right) \exp \left[-\frac{m \operatorname{Vol}\left(S^{3} \backslash \mathcal{K}\right)}{2 \pi} k\right] .
$$

From the above discussion, we expect the function $F_{m}(\mathcal{K})$ (for a prime knot $\mathcal{K}$ ) to grow as $k^{3 m}$ for large values of $k$ :

$$
F_{m}(\mathcal{K}) \sim C_{m}(\mathcal{K}) k^{3 m} .
$$

We first evaluate the values of $F_{m}(\mathcal{K})$ using (3.48) for sufficiently large values of $k$ and then use (3.49) to obtain the constants $C_{m}(\mathcal{K})$ numerically using the least-square fitting method in Mathematica. In section 4 , we present examples of prime knots up to six crossings, where $C_{m}(\mathcal{K})$ has been tabulated up to certain decimal places. With all the numerical results in hand, we propose the following:

Proposition. The large $k$ asymptotics of SO(3) Chern-Simons partition function of $M_{\mathcal{K}_{m}}$ is given as following:

$$
Z\left(M_{\mathcal{K}_{m}}\right)=\operatorname{Tr}\left[\sigma^{m}\left(\mathcal{K} \# T_{2,2}\right)\right] \sim C_{m}(\mathcal{K}) k^{3 m} \exp \left(\frac{m \operatorname{Vol}\left(S^{3} \backslash \mathcal{K}\right)}{2 \pi} k\right),
$$

where $C_{m}(\mathcal{K})$ is a knot-dependent constant. As a result, the Rényi entropies converge to a finite value in the limit of $k \rightarrow \infty$ and its value is determined by the constants $C_{m}(\mathcal{K})$ :

$$
\lim _{k \rightarrow \infty} \mathcal{R}_{m}=\frac{1}{1-m} \ln \left[\frac{C_{m}(\mathcal{K})}{C_{1}(\mathcal{K})^{m}}\right] .
$$

In the next section, we will present the numerical results validating our conjecture and proposal. We will restrict to prime knots up to six crossings.

\section{Numerical results for $\mathrm{SO}(3)$ group}

In this section, we will present the numerical results for all prime knots up to six crossings. A list of these knots, along with their hyperbolic volumes, are given in table 1. As discussed in the previous section, we obtain the constants $C_{m}(\mathcal{K})$ numerically for various values of $m$. We show the predicted variation of the function $C_{m}(\mathcal{K}) k^{3 m}$ and contrast it with the actual computed values of $F_{m}(\mathcal{K})$ from (3.48). We further list the proposed $k \rightarrow \infty$ limiting values 


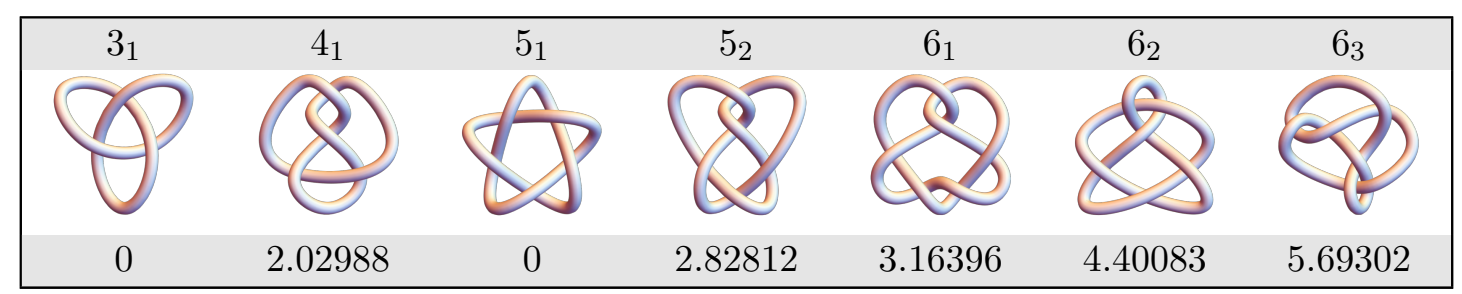

Table 1. List of all non-trivial prime knots up to six crossings. The first row of the table gives the notation of the knots according to the Rolfsen table [23]. The second row describes the knot diagram and the third row gives the hyperbolic volume of the knot complement.

of Rényi entropies obtained using $C_{m}(\mathcal{K})$ via (3.51). We justify these predicted limiting values by plotting the variation of Rényi entropies (calculated from (3.16)) with $k$ and showing that the plots tend to converge to the proposed values. For ease of presentation, a list of plots and tables for various knots are referred in the following:

\begin{tabular}{|c|c|c|c|c|c|}
\hline $\mathcal{K}$ & $C_{m}(\mathcal{K})$ & $C_{m}(\mathcal{K})$ vs $m$ & $F_{m}(\mathcal{K})$ vs $k$ & $\lim _{k \rightarrow \infty} \mathcal{R}_{m}$ & $\mathcal{R}_{m}$ vs $k$ \\
\hline $3_{1}$ & Table 2 & Figure 4 & Figure 5 & Table 3 & Figure 6 \\
$4_{1}$ & Table 6 & Figure 10 & Figure 11 & Table 7 & Figure 12 \\
$5_{1}$ & Table 4 & Figure 7 & Figure 8 & Table 5 & Figure 9 \\
$5_{2}$ & Table 8 & Figure 13 & Figure 14 & Table 9 & Figure 15 \\
$6_{1}$ & Table 10 & Figure 16 & Figure 17 & Table 11 & Figure 18 \\
$6_{2}$ & Table 12 & Figure 19 & Figure 20 & Table 13 & Figure 21 \\
$6_{3}$ & Table 14 & Figure 22 & Figure 23 & Table 15 & Figure 24 \\
\hline
\end{tabular}

We will take up the simplest torus knots in the following section to verify the results of previous section.

\subsection{Torus knots}

\subsubsection{Trefoil knot: $3_{1}$}

This is a torus knot with vanishing hyperbolic volume: $\operatorname{Vol}\left(S^{3} \backslash 3_{1}\right)=0$. The proposed form for the function $F_{m}$

$$
F_{m}\left(3_{1}\right)=C_{m}\left(3_{1}\right) k^{3 m}
$$

is computed using (3.48) for some sufficiently large values of $k$. In fact, the least-square method in Mathematica enables us to determine the constant $C_{m}\left(3_{1}\right)$ for various values of $m$. Some of these values are listed in table 2 and the variation of $\ln C_{m}\left(3_{1}\right)$ with $m$ is shown in figure 4 . In figure 5 , we plot the function $C_{m}\left(3_{1}\right) k^{3 m}$ and compare it with the numerical values of $F_{m}\left(3_{1}\right)$ obtained explicitly from (3.48). The predicted $k \rightarrow \infty$ values of the Rényi entropies associated with the state $\left|3_{1} \# T_{2,2}\right\rangle$ can be computed using (3.51) and we have listed some of these values in table 3 . We also show the variation of the Rényi entropies with $k$ in the plots in figure 6 . We see that these plots tend to converge to the predicted $k \rightarrow \infty$ values of the Rényi entropies. With this warm-up on trefoil, we present the numerical results for the five crossing torus knot. 


\begin{tabular}{|c|c|}
\hline$m$ & $C_{m}\left(3_{1}\right)$ \\
\hline 1 & $1.2508395500 \pm 0.0000141876$ \\
2 & $1.2724830787 \pm 0.0000133285$ \\
3 & $1.4267959549 \pm 0.0000139704$ \\
4 & $1.6058331673 \pm 0.0000151173$ \\
5 & $1.8076384699 \pm 0.0000167464$ \\
$\vdots$ & $\vdots$ \\
96 & $86270.3224528739 \pm 0.3000329135$ \\
97 & $97112.8266267165 \pm 0.3310750028$ \\
98 & $109318.0234964881 \pm 0.3652932337$ \\
99 & $123057.1771676096 \pm 0.4030095261$ \\
100 & $138523.0763324435 \pm 0.4445781686$ \\
\hline
\end{tabular}

Table 2. Values of $C_{m}\left(3_{1}\right)$.

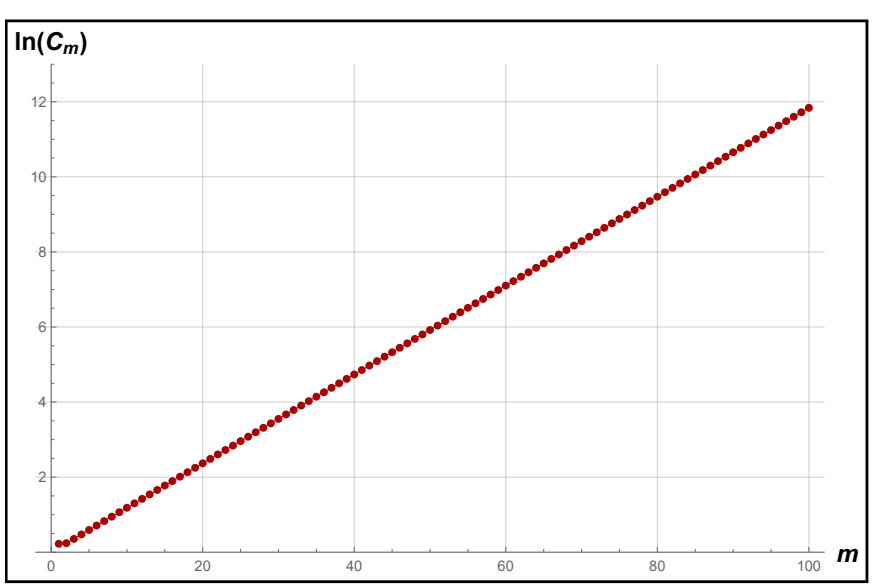

Figure 4. Variation of $\ln C_{m}\left(3_{1}\right)$ with $m$.
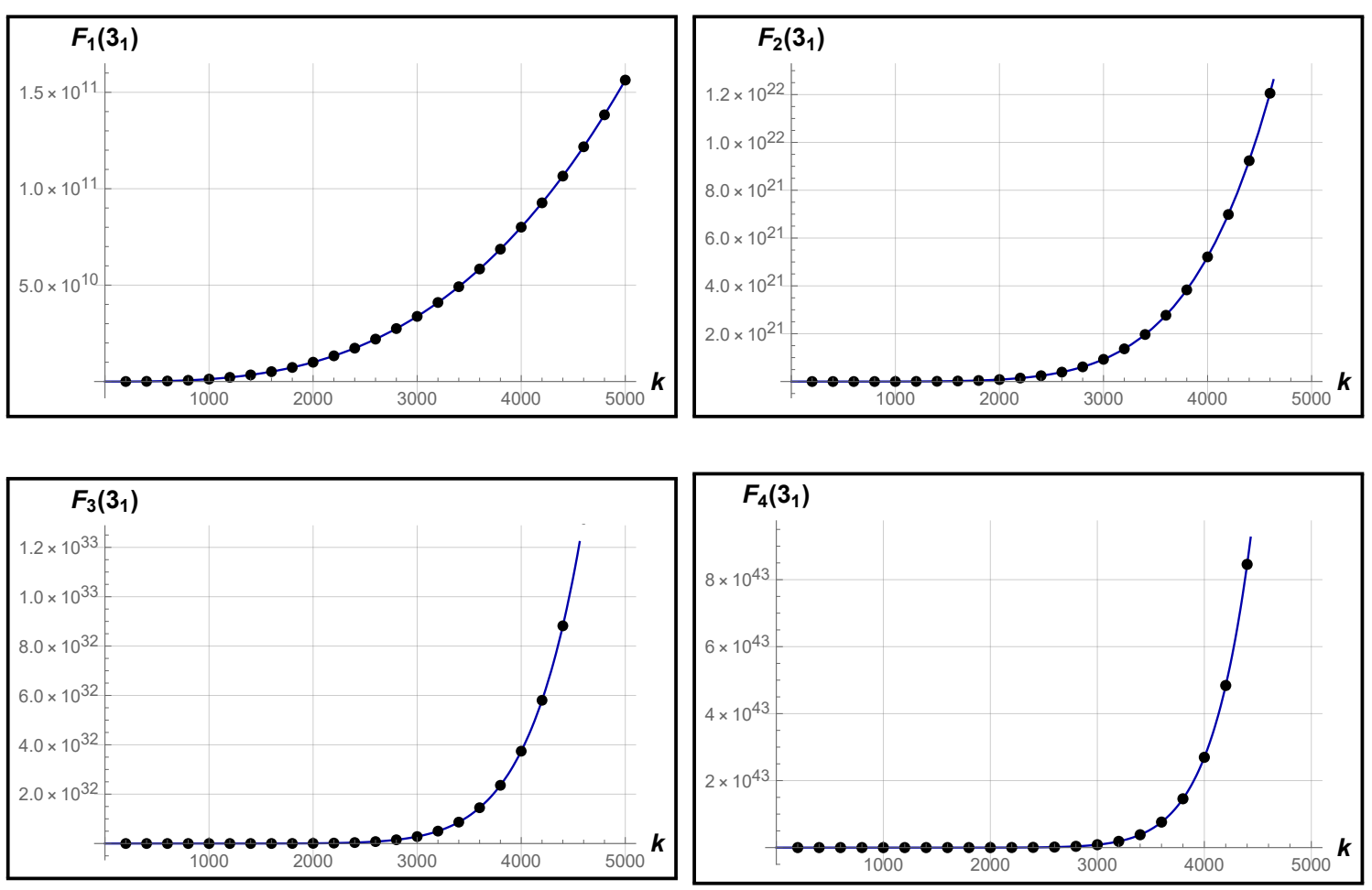

Figure 5. The variation of the function $F_{m}$ with $k$ for $3_{1}$ knot. The solid line denotes the function $C_{m} k^{3 m}$ with values of $C_{m}$ given in table 2 . The $\bullet$ indicates the values of $F_{m}$ obtained from explicit computations using (3.48). 


\begin{tabular}{|c|c|}
\hline$m$ & $\lim _{k \rightarrow \infty} \mathcal{R}_{m}$ \\
\hline 2 & $0.2066597601 \pm 0.0000249864$ \\
3 & $0.1580067791 \pm 0.0000177041$ \\
4 & $0.1405390447 \pm 0.0000154454$ \\
5 & $0.1317633872 \pm 0.0000143660$ \\
$\vdots$ & $\vdots$ \\
96 & $0.1065367978 \pm 0.0000114619$ \\
97 & $0.1065252390 \pm 0.0000114607$ \\
98 & $0.1065139185 \pm 0.0000114595$ \\
99 & $0.1065028290 \pm 0.0000114583$ \\
100 & $0.1064919634 \pm 0.0000114571$ \\
\hline
\end{tabular}

Table 3. The $k \rightarrow \infty$ values of the Rényi entropies associated with the state $\left|3_{1} \# T_{2,2}\right\rangle$.

\begin{tabular}{|c|c|}
\hline$m$ & $C_{m}\left(5_{1}\right)$ \\
\hline 1 & $1.0962198097 \pm 0.0000123719$ \\
2 & $1.0141454043 \pm 0.0000110043$ \\
3 & $1.0172196551 \pm 0.0000107028$ \\
4 & $1.0228630176 \pm 0.0000105694$ \\
5 & $1.0286308590 \pm 0.0000104842$ \\
$\vdots$ & $\vdots$ \\
96 & $1.7173551256 \pm 0.0000040805$ \\
97 & $1.7270567284 \pm 0.0000040224$ \\
98 & $1.7368131320 \pm 0.0000039648$ \\
99 & $1.7466246464 \pm 0.0000039076$ \\
100 & $1.7564915828 \pm 0.0000038509$ \\
\hline
\end{tabular}

Table 4. Values of $C_{m}\left(5_{1}\right)$.

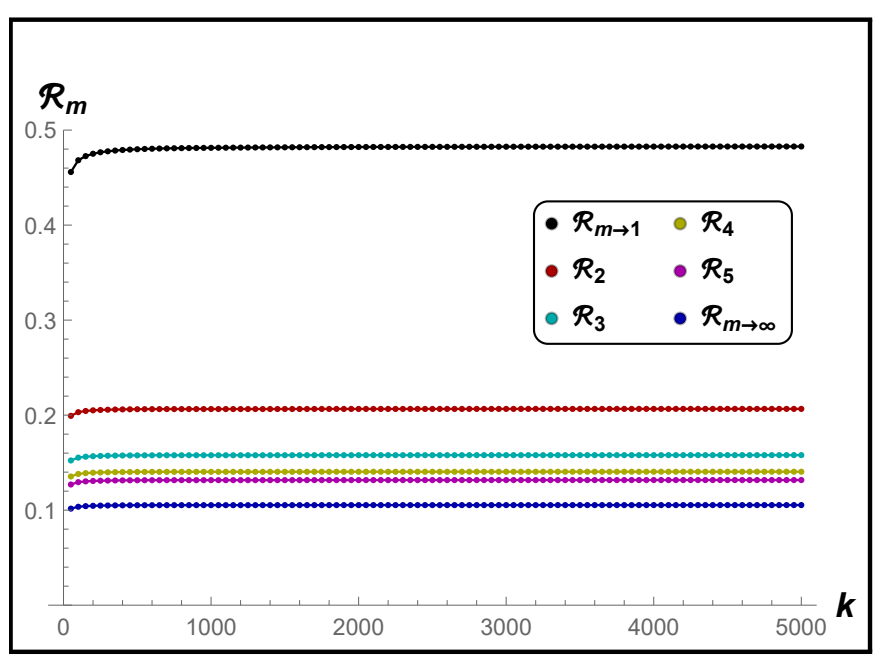

Figure 6. Variation of Rényi entropies for the state $\left|3_{1} \# T_{2,2}\right\rangle$ with $k$.

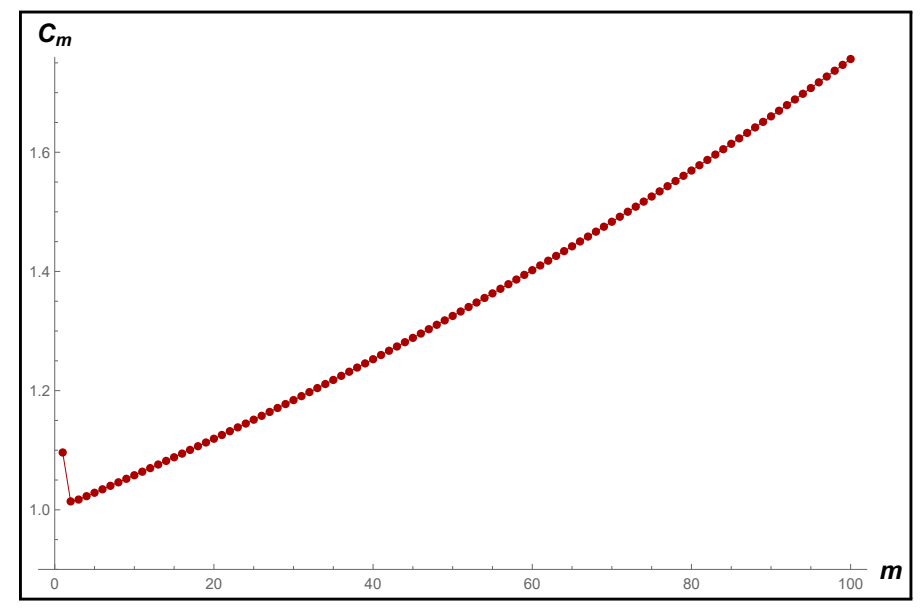

Figure 7. Variation of $C_{m}\left(5_{1}\right)$ with $m$.

\subsubsection{Solomon's Seal knot: $5_{1}$}

$5_{1}$ is also a torus knot with vanishing hyperbolic volume: $\operatorname{Vol}\left(S^{3} \backslash 5_{1}\right)=0$. Similar to our numerical methodology done for trefoil, we compute $F_{m}\left(5_{1}\right)$ (3.48) and using the proposed large $k$ form

$$
F_{m}\left(5_{1}\right)=C_{m}\left(5_{1}\right) k^{3 m},
$$

and least-square method in Mathematica, we obtained the constant $C_{m}\left(5_{1}\right)$ for various values of $m$. Some of these values are listed in table 4 and the variation of $C_{m}\left(5_{1}\right)$ with $m$ is shown in figure 7 . In figure 8 , we plot the function $C_{m}\left(5_{1}\right) k^{3 m}$ and compare it with the numerical values of $F_{m}\left(5_{1}\right)$ obtained explicitly from (3.48). The predicted $k \rightarrow \infty$ values of the Rényi entropies associated with the state $\left|5_{1} \# T_{2,2}\right\rangle$ can be computed using (3.51) and we 

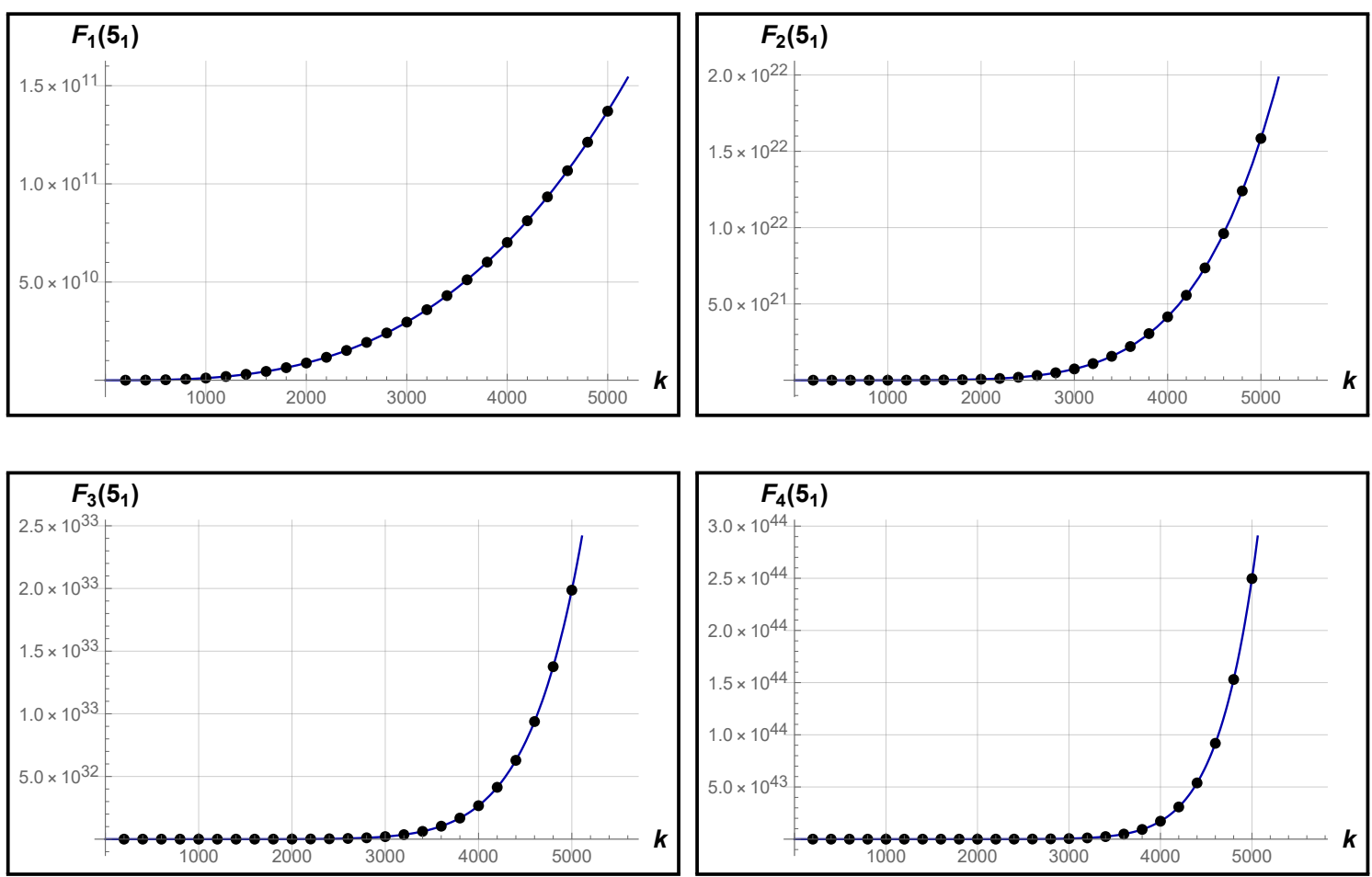

Figure 8. The variation of the function $F_{m}$ with $k$ for $5_{1}$ knot. The solid line denotes the function $C_{m} k^{3 m}$ with values of $C_{m}$ given in table 4 . The $\bullet$ indicates the numerical values of $F_{m}$ obtained from explicit computations using (3.48).

have listed some of these values in table 5. We also show the variation of the Rényi entropies with $k$ in the plots in figure 9 . We see that these plots tend to converge to the predicted $k \rightarrow \infty$ values of the Rényi entropies. We have also done similar numerical validation for other knots with non-trivial hyperbolic volume which we present in the following subsection.

\subsection{Non-torus knots}

\subsubsection{Figure-eight knot: $4_{1}$}

The lowest crossing non-torus knot is the figure-eight knot $4_{1}$ with the hyperbolic volume $\operatorname{Vol}\left(S^{3} \backslash 4_{1}\right)=2.029883212819307$. Using $F_{m}\left(4_{1}\right)$ (3.48) and the following proposed large $k$ form,

$$
F_{m}\left(4_{1}\right)=C_{m}\left(4_{1}\right) k^{3 m}
$$

the constant $C_{m}\left(4_{1}\right)$ was deduced using the least-square method for various values of $m$. Some of these values are listed in table 6 and the variation of $\ln C_{m}\left(4_{1}\right)$ with $m$ is shown in figure 10. In figure 11, we plot the function $C_{m}\left(4_{1}\right) k^{3 m}$ and compare it with the numerical values of $F_{m}\left(4_{1}\right)$ obtained explicitly from (3.48). The predicted $k \rightarrow \infty$ values of the Rényi entropies associated with the state $\left|4_{1} \# T_{2,2}\right\rangle$ can be computed using (3.51) and we have listed some of these values in table 7. We also show the variation of the Rényi entropies with $k$ in the plots in figure 12 . We see that these plots tend to converge to the predicted $k \rightarrow \infty$ values 


\begin{tabular}{|c|c|}
\hline$m$ & $\lim _{k \rightarrow \infty} \mathcal{R}_{m}$ \\
\hline 2 & $0.1696891578 \pm 0.0000250446$ \\
3 & $0.1292650485 \pm 0.0000177275$ \\
4 & $0.1149551079 \pm 0.0000154371$ \\
5 & $0.1077774922 \pm 0.0000143357$ \\
$\vdots$ & $\vdots$ \\
96 & $0.0871422756 \pm 0.0000114048$ \\
97 & $0.0871328193 \pm 0.0000114036$ \\
98 & $0.0871235580 \pm 0.0000114023$ \\
99 & $0.0871144857 \pm 0.0000114012$ \\
100 & $0.0871055967 \pm 0.0000114000$ \\
\hline
\end{tabular}

Table 5. The $k \rightarrow \infty$ values of the Rényi entropies associated with the state $\left|5_{1} \# T_{2,2}\right\rangle$.

\begin{tabular}{|c|c|}
\hline$m$ & $C_{m}\left(4_{1}\right)$ \\
\hline 1 & $2.5957570692 \pm 0.0001620425$ \\
2 & $6.2495177996 \pm 0.0004659030$ \\
3 & $15.6076018833 \pm 0.0011272905$ \\
4 & $38.9986179208 \pm 0.0027607882$ \\
5 & $97.4465517130 \pm 0.0067868268$ \\
6 & $243.4918590302 \pm 0.0167002962$ \\
7 & $608.4194021638 \pm 0.0410857092$ \\
8 & $1520.2754646304 \pm 0.1009983598$ \\
9 & $3798.7623179296 \pm 0.2480046124$ \\
10 & $9492.1051075026 \pm 0.6082069793$ \\
\hline
\end{tabular}

Table 6. Values of $C_{m}\left(4_{1}\right)$.

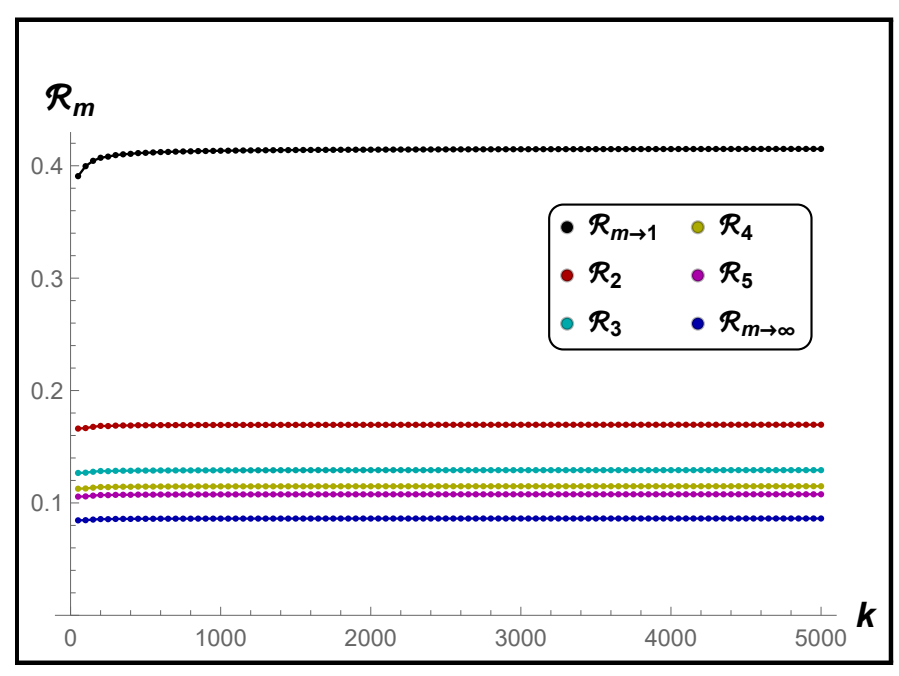

Figure 9. Variation of Rényi entropies for the state $\left|5_{1} \# T_{2,2}\right\rangle$ with $k$.

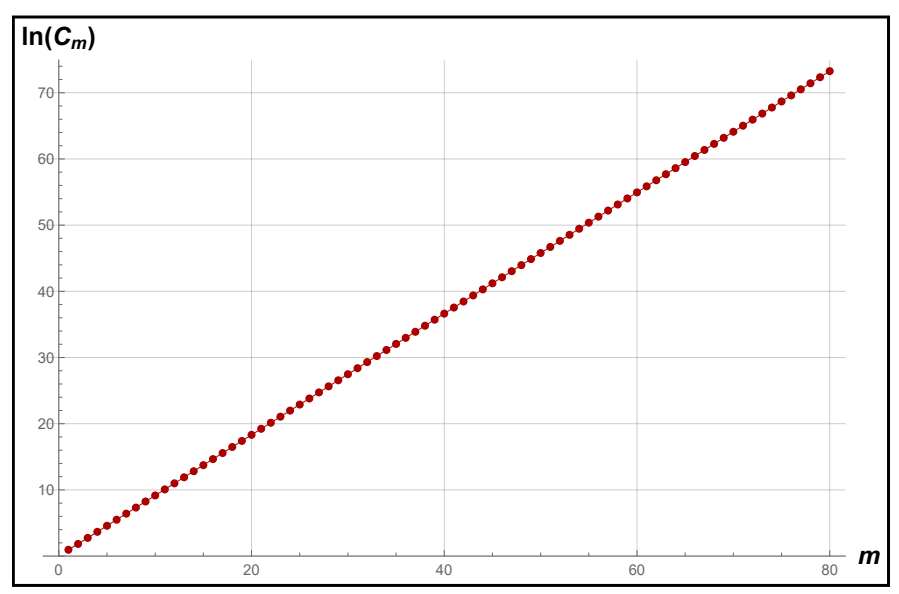

Figure 10. Variation of $\ln C_{m}\left(4_{1}\right)$ with $m$.

of the Rényi entropies. We present similar analysis and our numerical results for the five crossing non-torus knot in the following section to validate our conjecture and the proposal.

\subsubsection{Three-twist knot: $\mathbf{5}_{2}$}

$F_{m}\left(5_{2}\right)$ for this five crossing non-torus knot, whose knot complement volume is $\operatorname{Vol}\left(S^{3} \backslash 5_{2}\right)=$ 2.828122088330783 , is computed using (3.48). Then, from the proposed large $k$ form

$$
F_{m}\left(5_{2}\right)=C_{m}\left(5_{2}\right) k^{3 m}
$$

the constant $C_{m}\left(5_{2}\right)$ is determined using the least-square method for various values of $m$. Some of these values are listed in table 8 and the variation of $\ln C_{m}\left(5_{2}\right)$ with $m$ is shown in figure 13. In figure 14, we plot the function $C_{m}\left(5_{2}\right) k^{3 m}$ and compare it with the numerical values of $F_{m}\left(5_{2}\right)$ obtained explicitly from (3.48). The predicted $k \rightarrow \infty$ values of the Rényi 

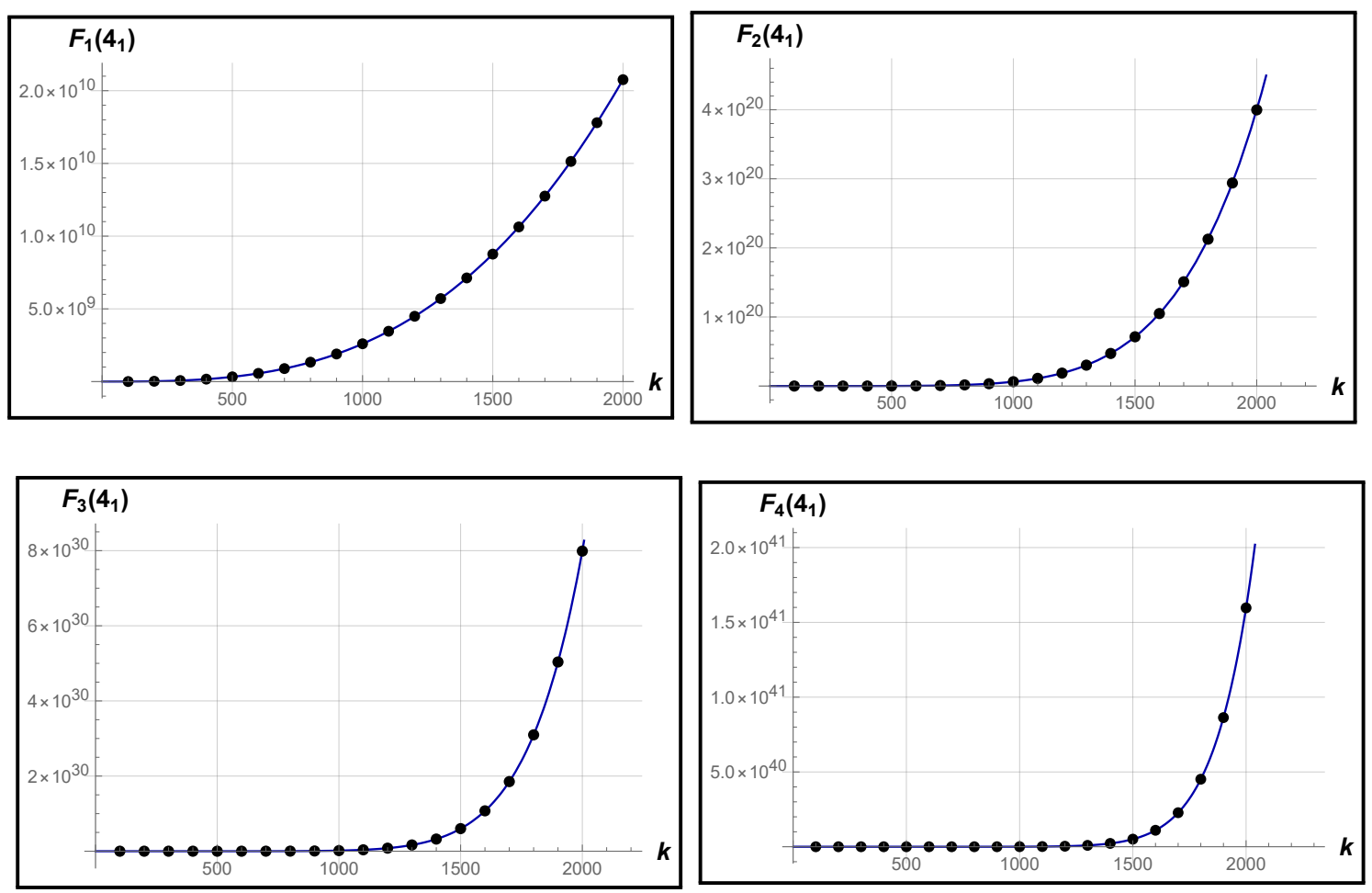

Figure 11. The variation of the function $F_{m}$ with $k$ for $4_{1}$ knot. The solid line denotes the function $C_{m} k^{3 m}$ with values of $C_{m}$ given in table 6 . The $\bullet$ indicates the numerical values of $F_{m}$ obtained from explicit computations.

\begin{tabular}{|c|c|}
\hline$m$ & $\lim _{k \rightarrow \infty} \mathcal{R}_{m}$ \\
\hline 2 & $0.0752521224 \pm 0.0001454157$ \\
3 & $0.0569382755 \pm 0.0001003615$ \\
4 & $0.0506622182 \pm 0.0000865149$ \\
5 & $0.0475217594 \pm 0.0000799514$ \\
6 & $0.0456371569 \pm 0.0000761567$ \\
7 & $0.0443805098 \pm 0.0000736947$ \\
8 & $0.0434827000 \pm 0.0000719724$ \\
9 & $0.0428091692 \pm 0.0000707017$ \\
10 & $0.0422851628 \pm 0.0000697266$ \\
\hline
\end{tabular}

Table 7. The $k \rightarrow \infty$ values of the Rényi entropies associated with the state $\left|4_{1} \# T_{2,2}\right\rangle$.

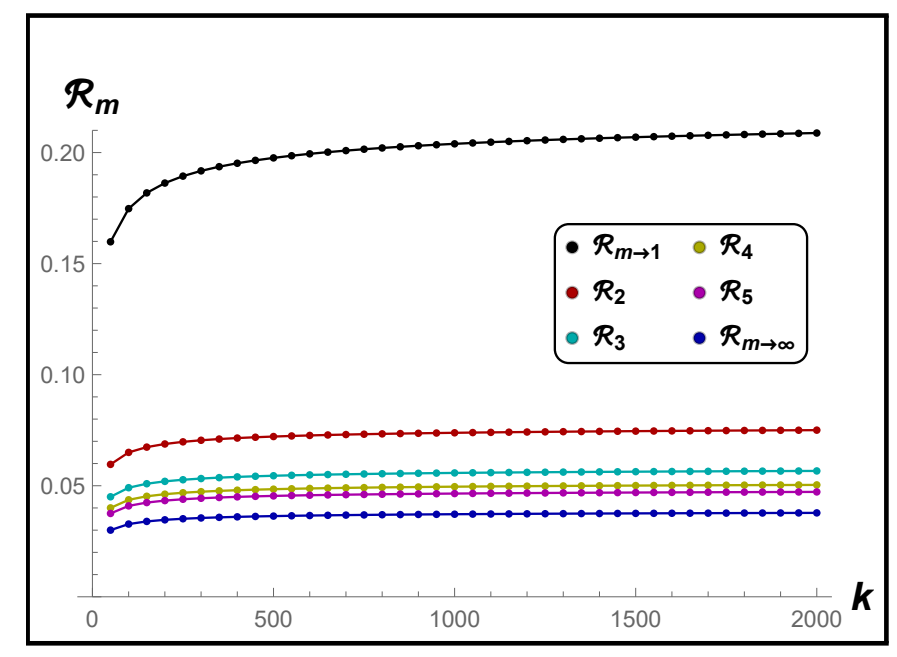

Figure 12. Variation of Rényi entropies for the state $\left|4_{1} \# T_{2,2}\right\rangle$ with $k$. 


\begin{tabular}{|c|c|}
\hline$m$ & $C_{m}\left(5_{2}\right)$ \\
\hline 1 & $4.5156047311 \pm 0.0080192291$ \\
2 & $19.522561281 \pm 0.0323263046$ \\
3 & $85.809660629 \pm 0.1375626362$ \\
4 & $377.22537837 \pm 0.5933078608$ \\
5 & $1658.35734226 \pm 2.5705101499$ \\
6 & $7290.66518025 \pm 11.154379776$ \\
7 & $32052.9326226 \pm 48.419257460$ \\
8 & $140922.2991482 \pm 210.12155723$ \\
9 & $619587.6221496 \pm 911.29217860$ \\
10 & $2724184.491914 \pm 3949.0717321$ \\
\hline
\end{tabular}

Table 8. Values of $C_{m}\left(5_{2}\right)$.

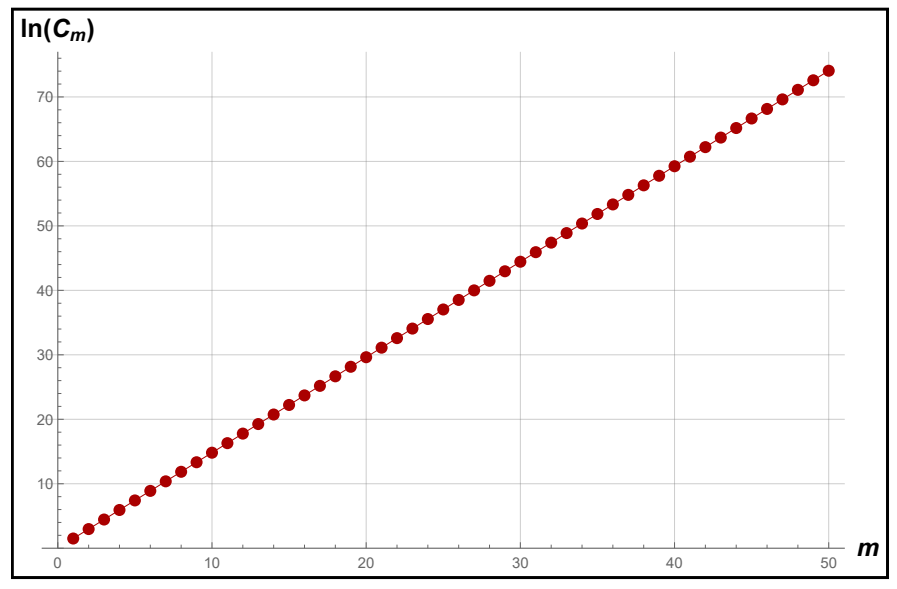

Figure 13. Variation of $\ln C_{m}\left(5_{2}\right)$ with $m$.
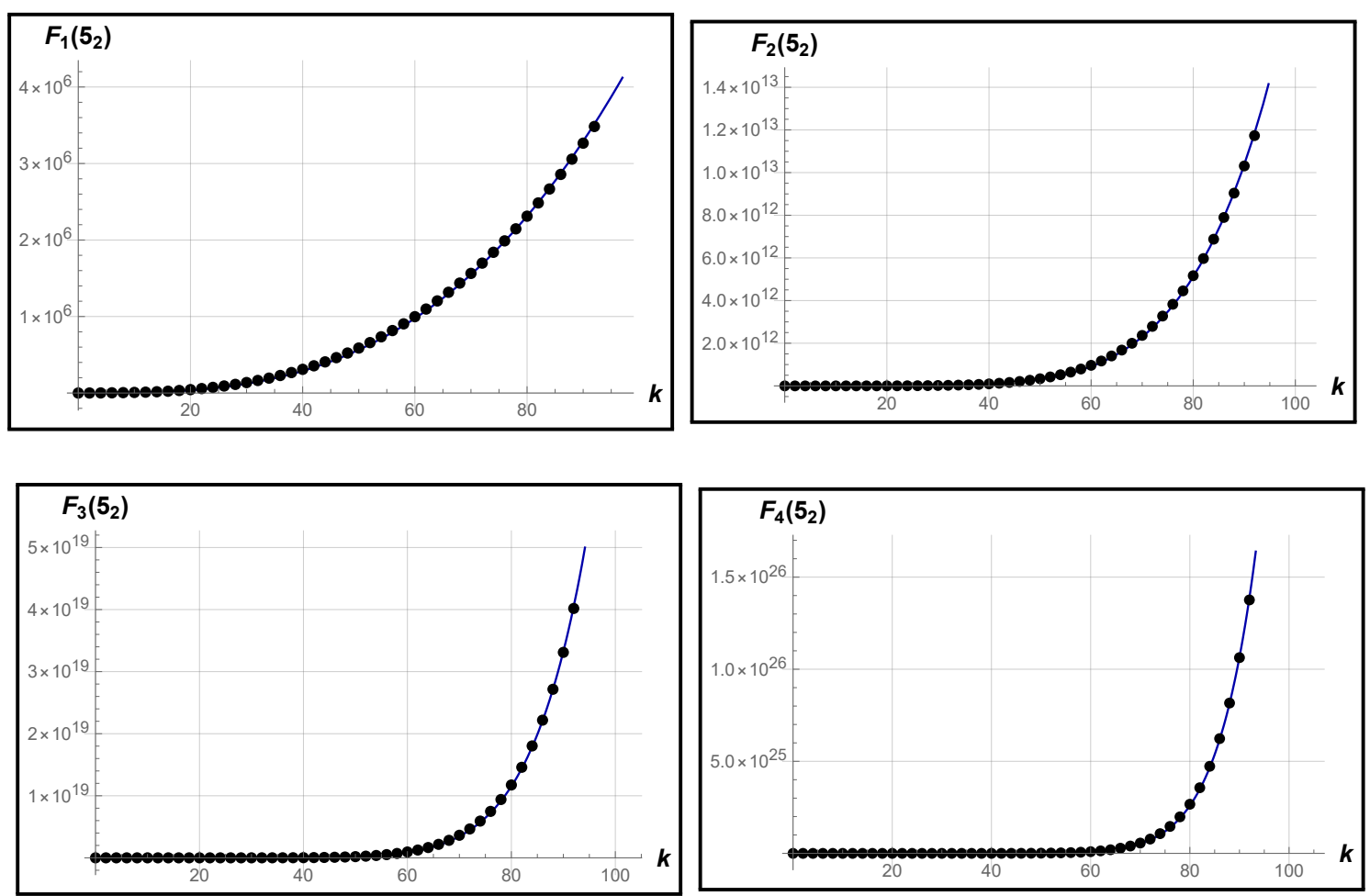

Figure 14. The variation of the function $F_{m}$ with $k$ for $5_{2}$ knot. The solid line denotes the function $C_{m} k^{3 m}$ with values of $C_{m}$ given in table 8 . The $\bullet$ indicates the numerical values of $F_{m}$ obtained from explicit computations. 


\begin{tabular}{|c|c|}
\hline$m$ & $\lim _{k \rightarrow \infty} \mathcal{R}_{m}$ \\
\hline 2 & $0.0435074467 \pm 0.0039188003$ \\
3 & $0.0352428767 \pm 0.0027818216$ \\
4 & $0.0324378784 \pm 0.0024252028$ \\
5 & $0.0310281855 \pm 0.0022534345$ \\
6 & $0.0301769261 \pm 0.0021529270$ \\
7 & $0.0296049755 \pm 0.0020871156$ \\
8 & $0.0291927116 \pm 0.0020407385$ \\
9 & $0.0288803294 \pm 0.0020063206$ \\
10 & $0.0286346099 \pm 0.0019797771$ \\
\hline
\end{tabular}

Table 9. The $k \rightarrow \infty$ values of the Rényi entropies associated with the state $\left|5_{2} \# T_{2,2}\right\rangle$.

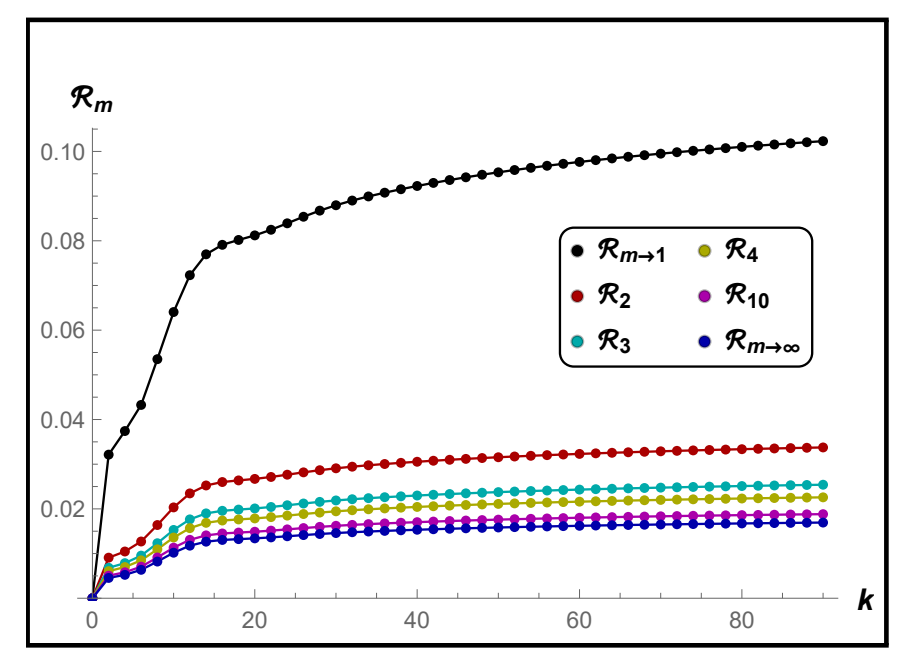

Figure 15. Variation of Rényi entropies for the state $\left|5_{2} \# T_{2,2}\right\rangle$ with $k$.

entropies associated with the state $\left|5_{2} \# T_{2,2}\right\rangle$ can be computed using (3.51) and we have listed some of these values in table 9 . We also show the variation of the Rényi entropies with $k$ in the plots in figure 15 . We see that these plots tend to converge to the predicted $k \rightarrow \infty$ values of the Rényi entropies. In the rest of this section, we address six crossing non-torus knots to verify our conjecture and proposal.

\subsubsection{Stevedore knot: $6_{1}$}

This is a non-torus knot whose knot complement volume is $\operatorname{Vol}\left(S^{3} \backslash 6_{1}\right)=3.16396322888$. In fact, computing $F_{m}\left(6_{1}\right)$ (3.48) and relating to the form

$$
F_{m}\left(6_{1}\right) \equiv \frac{\operatorname{Tr}\left[\sigma^{m}\left(6_{1} \# T_{2,2}\right)\right]}{\exp \left(\frac{m \operatorname{Vol}\left(S^{3} \backslash 6_{1}\right)}{2 \pi} k\right)}=C_{m}\left(6_{1}\right) k^{3 m},
$$

and using the least-square method, we computed $C_{m}\left(6_{1}\right)$ for various values of $m$. Some of these values are listed in table 10 and the variation of $\ln C_{m}\left(6_{1}\right)$ with $m$ is shown in figure 16. In figure 17, we plot the function $C_{m}\left(6_{1}\right) k^{3 m}$ and compare it with the numerical values of $F_{m}\left(6_{1}\right)$ obtained explicitly from (3.48). The predicted $k \rightarrow \infty$ values of the Rényi entropies associated with the state $\left|6_{1} \# T_{2,2}\right\rangle$ can be computed using (3.51) and we have listed some of these values in table 11. We also show the variation of the Rényi entropies with $k$ in the plots in figure 18. We see that these plots tend to converge to the predicted $k \rightarrow \infty$ values of the Rényi entropies.

\subsection{4 $\quad 6_{2}$ knot}

The hyperbolic volume is $\operatorname{Vol}\left(S^{3} \backslash 6_{2}\right)=4.400832516$ for the complement of the non-torus knot $6_{2}$. We computed $F_{m}\left(6_{2}\right)(3.48)$ and compared with the proposed form

$$
F_{m}\left(6_{2}\right) \equiv \frac{\operatorname{Tr}\left[\sigma^{m}\left(6_{2} \# T_{2,2}\right)\right]}{\exp \left(\frac{m \operatorname{Vol}\left(S^{3} \backslash 6_{2}\right)}{2 \pi} k\right)}=C_{m}\left(6_{2}\right) k^{3 m}
$$




\begin{tabular}{|c|c|}
\hline$m$ & $C_{m}\left(6_{1}\right)$ \\
\hline 1 & $4.7923232325 \pm 0.0030931604$ \\
2 & $22.3923919843 \pm 0.0135258863$ \\
3 & $105.7984752566 \pm 0.0616708792$ \\
4 & $499.9052116194 \pm 0.2842710891$ \\
5 & $2362.131219566 \pm 1.3135290735$ \\
6 & $11161.64299789 \pm 6.0672497245$ \\
7 & $52742.37636985 \pm 27.982406758$ \\
8 & $249228.8880632 \pm 128.78990106$ \\
9 & $1177724.7827384 \pm 591.37837422$ \\
10 & $5565389.9109037 \pm 2708.8286274$ \\
\hline
\end{tabular}

Table 10. Values of $C_{m}\left(6_{1}\right)$.

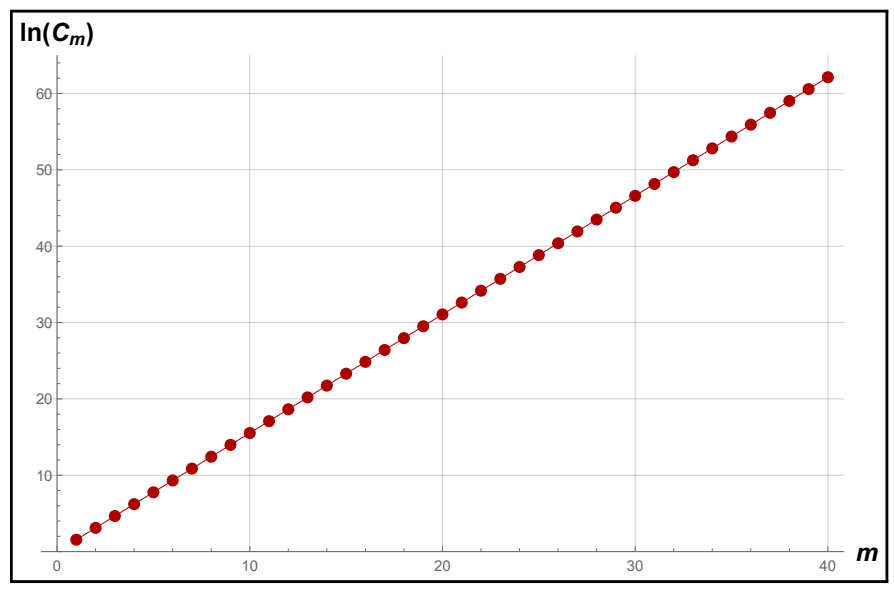

Figure 16. Variation of $\ln C_{m}\left(6_{1}\right)$ with $m$.
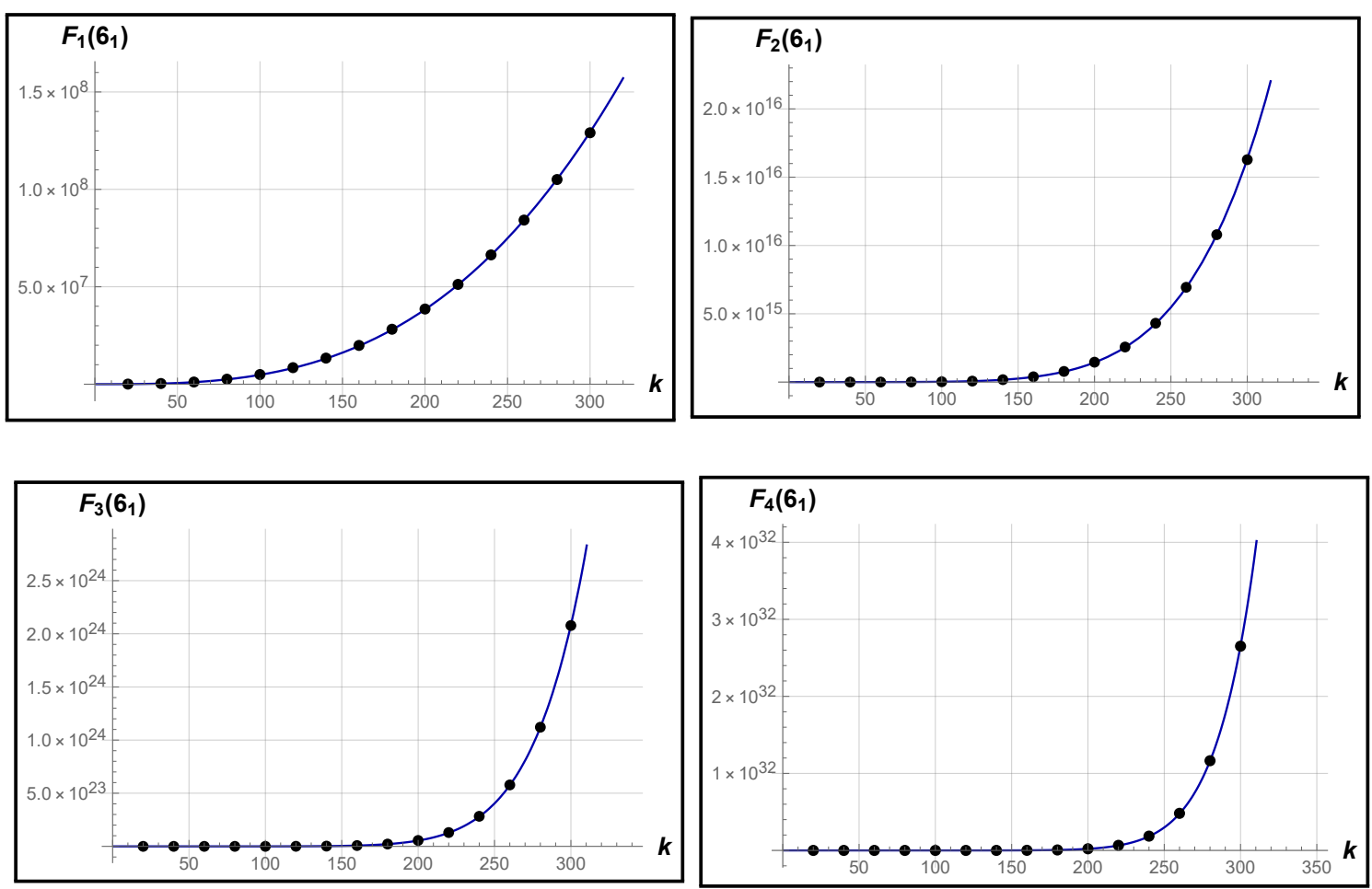

Figure 17. The variation of the function $F_{m}$ with $k$ for $6_{1}$ knot. The solid line denotes the function $C_{m} k^{3 m}$ with values of $C_{m}$ given in table 10 . The $\bullet$ indicates the numerical values of $F_{m}$ obtained from explicit computations. 


\begin{tabular}{|c|c|}
\hline$m$ & $\lim _{k \rightarrow \infty} \mathcal{R}_{m}$ \\
\hline 2 & $0.0253093645 \pm 0.0014252152$ \\
3 & $0.0197549127 \pm 0.0010110794$ \\
4 & $0.0178809135 \pm 0.0008812152$ \\
5 & $0.0169392514 \pm 0.0008186904$ \\
6 & $0.0163706838 \pm 0.0007821215$ \\
7 & $0.0159887767 \pm 0.0007581881$ \\
8 & $0.0157136437 \pm 0.0007413313$ \\
9 & $0.0155053518 \pm 0.0007288286$ \\
10 & $0.0153417228 \pm 0.0007191926$ \\
\hline
\end{tabular}

Table 11. The $k \rightarrow \infty$ values of the Rényi entropies associated with the state $\left|6_{1} \# T_{2,2}\right\rangle$.

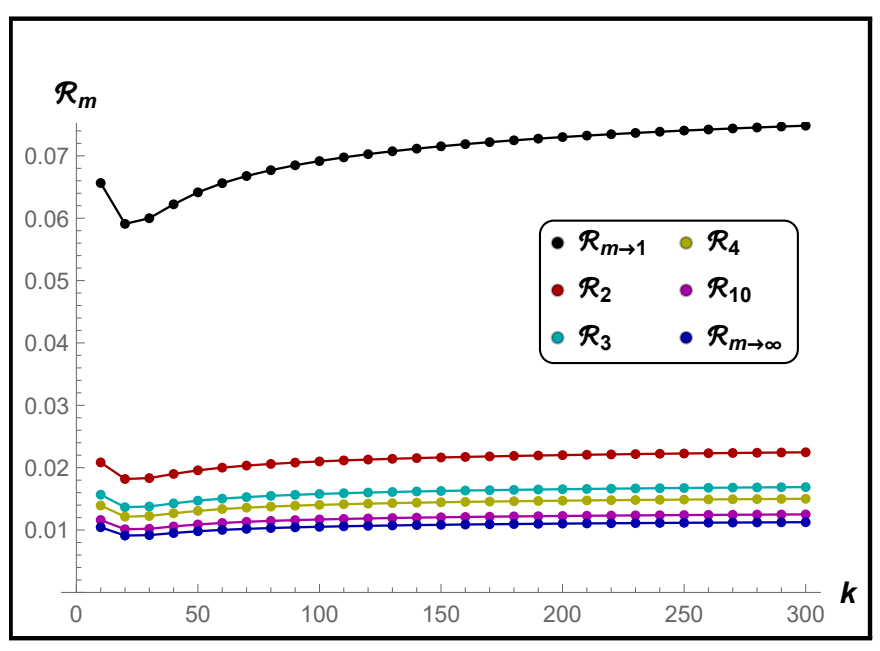

Figure 18. Variation of Rényi entropies for the state $\left|6_{1} \# T_{2,2}\right\rangle$ with $k$.

\begin{tabular}{|c|c|}
\hline$m$ & $C_{m}\left(6_{2}\right)$ \\
\hline 1 & $7.2078662291 \pm 0.0114864102$ \\
2 & $50.9252866533 \pm 0.0745214114$ \\
3 & $362.4041343538 \pm 0.5060376066$ \\
4 & $2579.2833524324 \pm 3.4585013119$ \\
5 & $18358.617056 \pm 23.58889$ \\
6 & $130681.2420 \pm 160.1351$ \\
7 & $930284.933 \pm 1080.959$ \\
8 & $6622851.572 \pm 7253.540$ \\
9 & $47151678.319 \pm 48386.251$ \\
10 & $335713848.188 \pm 320930.180$ \\
\hline
\end{tabular}

Table 12. Values of $C_{m}\left(6_{2}\right)$.

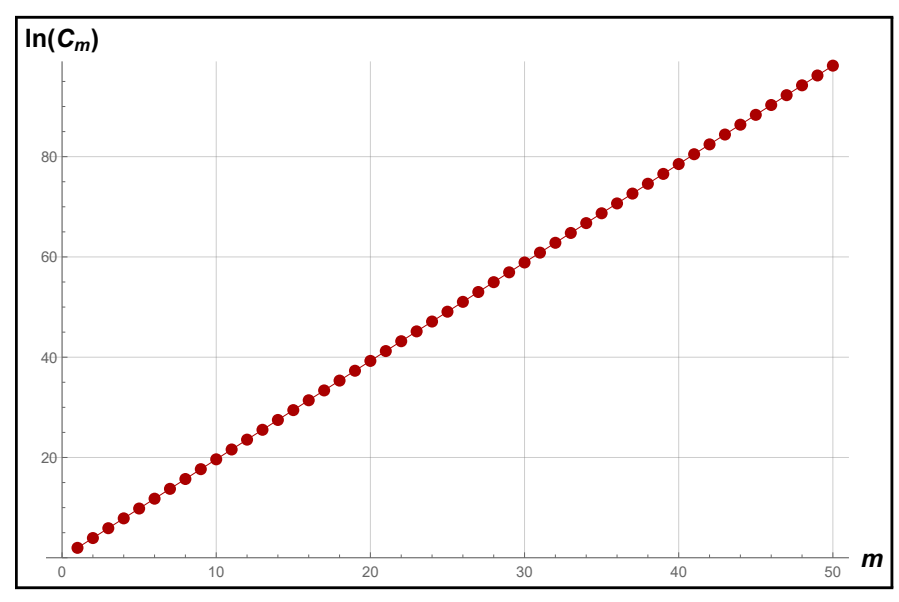

Figure 19. Variation of $\ln C_{m}\left(6_{2}\right)$ with $m$.

to deduce $C_{m}\left(6_{2}\right)$ for various values of $m$ using least-square method. Some of these values are listed in table 12 and the variation of $\ln C_{m}\left(6_{2}\right)$ with $m$ is shown in figure 19. In figure 20, we plot the function $C_{m}\left(6_{2}\right) k^{3 m}$ and compare it with the numerical values of $F_{m}\left(6_{2}\right)$ obtained explicitly from (3.48). The predicted $k \rightarrow \infty$ values of the Rényi entropies associated with the state $\left|6_{2} \# T_{2,2}\right\rangle$ can be computed using (3.51) and we have listed some of these values in table 13. We also show the variation of the Rényi entropies with $k$ in the plots in figure 21 . We see that these plots tend to converge to the predicted $k \rightarrow \infty$ values of the Rényi entropies. 

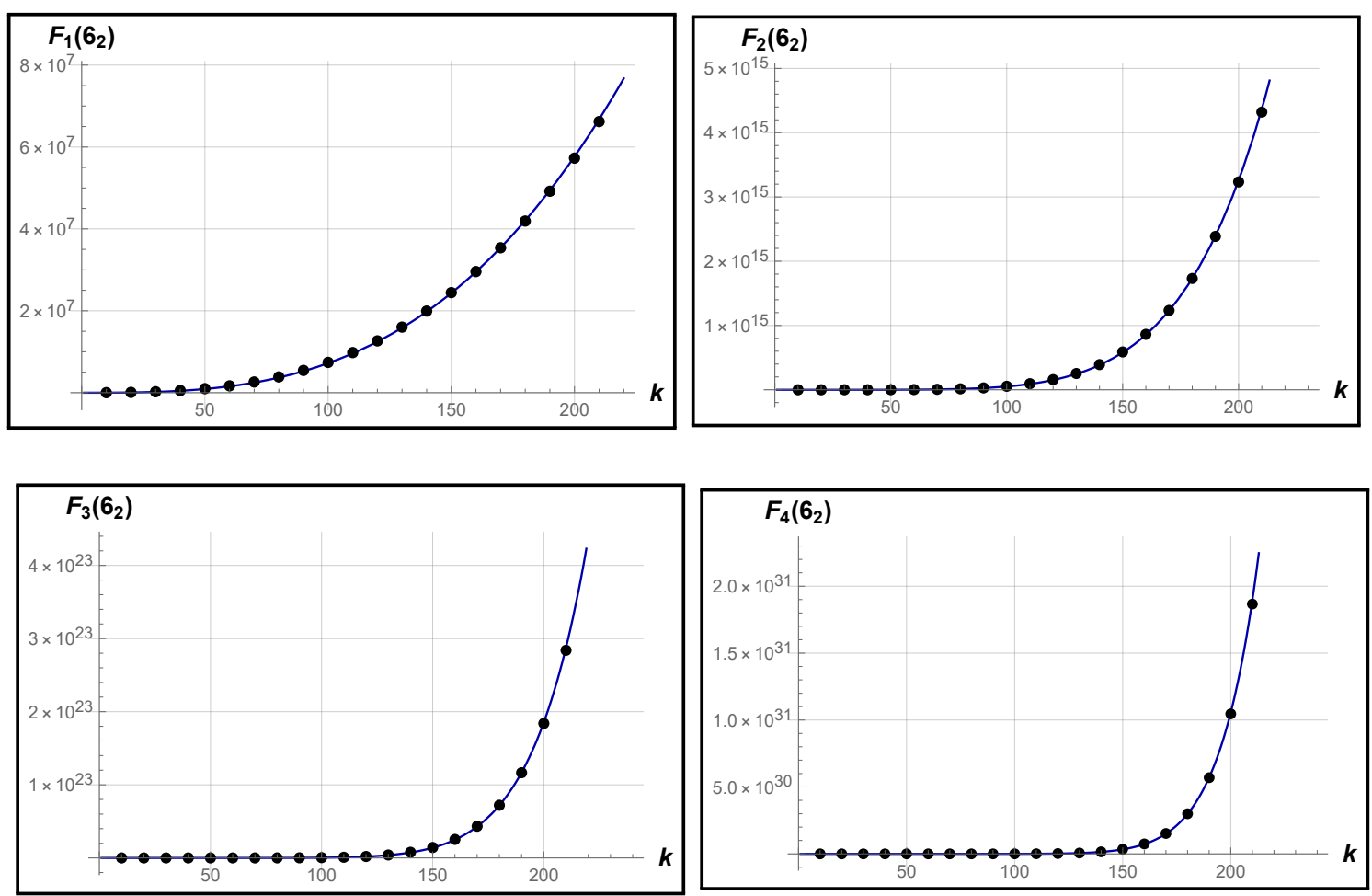

Figure 20. The variation of the function $F_{m}$ with $k$ for $6_{2}$ knot. The solid line denotes the function $C_{m} k^{3 m}$ with values of $C_{m}$ given in table 12 . The $\bullet$ indicates the numerical values of $F_{m}$ obtained from explicit computations.

\begin{tabular}{|c|c|}
\hline$m$ & $\lim _{k \rightarrow \infty} \mathcal{R}_{m}$ \\
\hline 2 & $0.0199863319 \pm 0.0035070715$ \\
3 & $0.0163794510 \pm 0.0024902620$ \\
4 & $0.0151416590 \pm 0.0021712926$ \\
5 & $0.0145026174 \pm 0.0020177258$ \\
6 & $0.0141042798 \pm 0.0019279527$ \\
7 & $0.0138274220 \pm 0.0018692517$ \\
8 & $0.0136210151 \pm 0.0018279583$ \\
9 & $0.0134595614 \pm 0.0017973760$ \\
10 & $0.0133288780 \pm 0.0017738427$ \\
\hline
\end{tabular}

Table 13. The $k \rightarrow \infty$ values of the Rényi entropies associated with the state $\left|6_{2} \# T_{2,2}\right\rangle$.

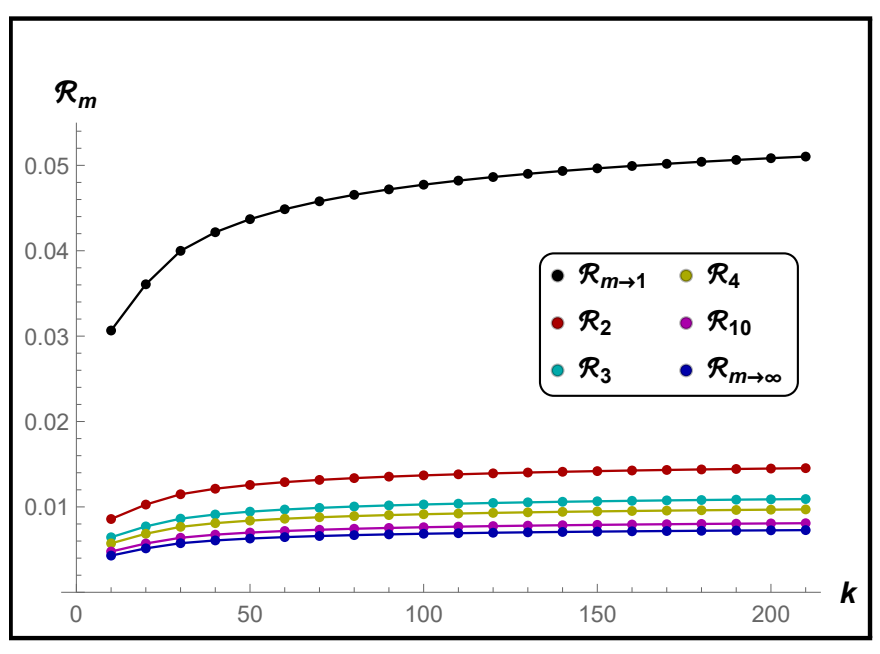

Figure 21. Variation of Rényi entropies for the state $\left|6_{2} \# T_{2,2}\right\rangle$ with $k$. 


\begin{tabular}{|c|c|}
\hline$m$ & $C_{m}\left(6_{3}\right)$ \\
\hline 1 & $9.5970632893 \pm 0.0185978565$ \\
2 & $90.554839418 \pm 0.160095501$ \\
3 & $858.76989699 \pm 1.44999102$ \\
4 & $8144.95594531 \pm 13.23524518$ \\
5 & $77257.4532157 \pm 120.6783625$ \\
6 & $732873.448104 \pm 1096.079889$ \\
7 & $6952667.48358 \pm 9906.38029$ \\
8 & $65963622.8502 \pm 89061.0909$ \\
9 & $625870830.613 \pm 796416.850$ \\
10 & $5938663602.54 \pm 7084757.98$ \\
\hline
\end{tabular}

Table 14. Values of $C_{m}\left(6_{3}\right)$.

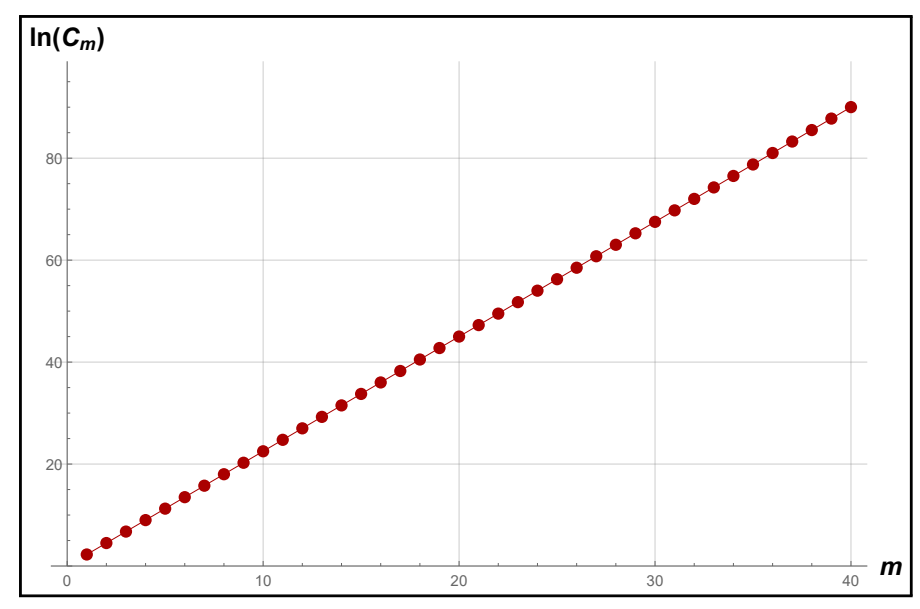

Figure 22. Variation of $\ln C_{m}\left(6_{3}\right)$ with $m$.

\subsection{5 $\quad 6_{3}$ knot}

Finally, we address similar analysis for $6_{3}$ knot whose knot complement volume is $\operatorname{Vol}\left(S^{3} \backslash 6_{3}\right)=5.69302109$. Thus, working out $F_{m}\left(6_{3}\right)$ (3.48) and comparing with

$$
F_{m}\left(6_{3}\right) \equiv \frac{\operatorname{Tr}\left[\sigma^{m}\left(6_{3} \# T_{2,2}\right)\right]}{\exp \left(\frac{m \operatorname{Vol}\left(S^{3} \backslash 6_{3}\right)}{2 \pi} k\right)}=C_{m}\left(6_{3}\right) k^{3 m},
$$

the constant $C_{m}\left(6_{3}\right)$ can be deduced for various values of $m$ using the least-square method. Some of these values are listed in table 14 and the variation of $\ln C_{m}\left(6_{3}\right)$ with $m$ is shown in figure 22. In figure 23, we plot the function $C_{m}\left(6_{3}\right) k^{3 m}$ and compare it with the numerical values of $F_{m}\left(6_{3}\right)$ obtained explicitly from (3.48). The predicted $k \rightarrow \infty$ values of the Rényi entropies associated with the state $\left|6_{3} \# T_{2,2}\right\rangle$ can be computed using (3.51) and we have listed some of these values in table 15 . We also show the variation of the Rényi entropies with $k$ in the plots in figure 24 . We see that these plots tend to converge to the predicted $k \rightarrow \infty$ values of the Rényi entropies.

These numerical analysis and plots for all the prime knots up to six crossings indeed validate our conjecture and proposal within $\mathrm{SO}(3)$ Chern-Simons theory.

\section{Conclusion}

In this paper, our primary goal was to capture the geometrical features of the three-manifolds from the study of entanglement properties of bi-partite states. Particularly, we focused on the Chern-Simons theory to study the topological entanglement between two torus boundaries of a three-manifold $S^{3} \backslash \mathcal{L}$ (the link complement of a two-component link $\mathcal{L}$ ). Interestingly, for a class of two-component links viewed as a connected sum of a prime knot $\mathcal{K}$ and the Hopf link $\left(\mathcal{L}=\mathcal{K} \# T_{2,2}\right)$, the probability amplitudes of the state $\left|\mathcal{K} \# T_{2,2}\right\rangle$ are proportional to the quantum invariants of the knot $\mathcal{K}$. Thus the trace of the reduced density matrix (1.3) is almost similar to the Turaev-Viro invariant $[16,17]$ of $S^{3} \backslash \mathcal{K}$. This motivated us to study the reduced density matrices for this class of two-component links for both $\mathrm{SU}(2)$ and $\mathrm{SO}(3)$ gauge groups. 

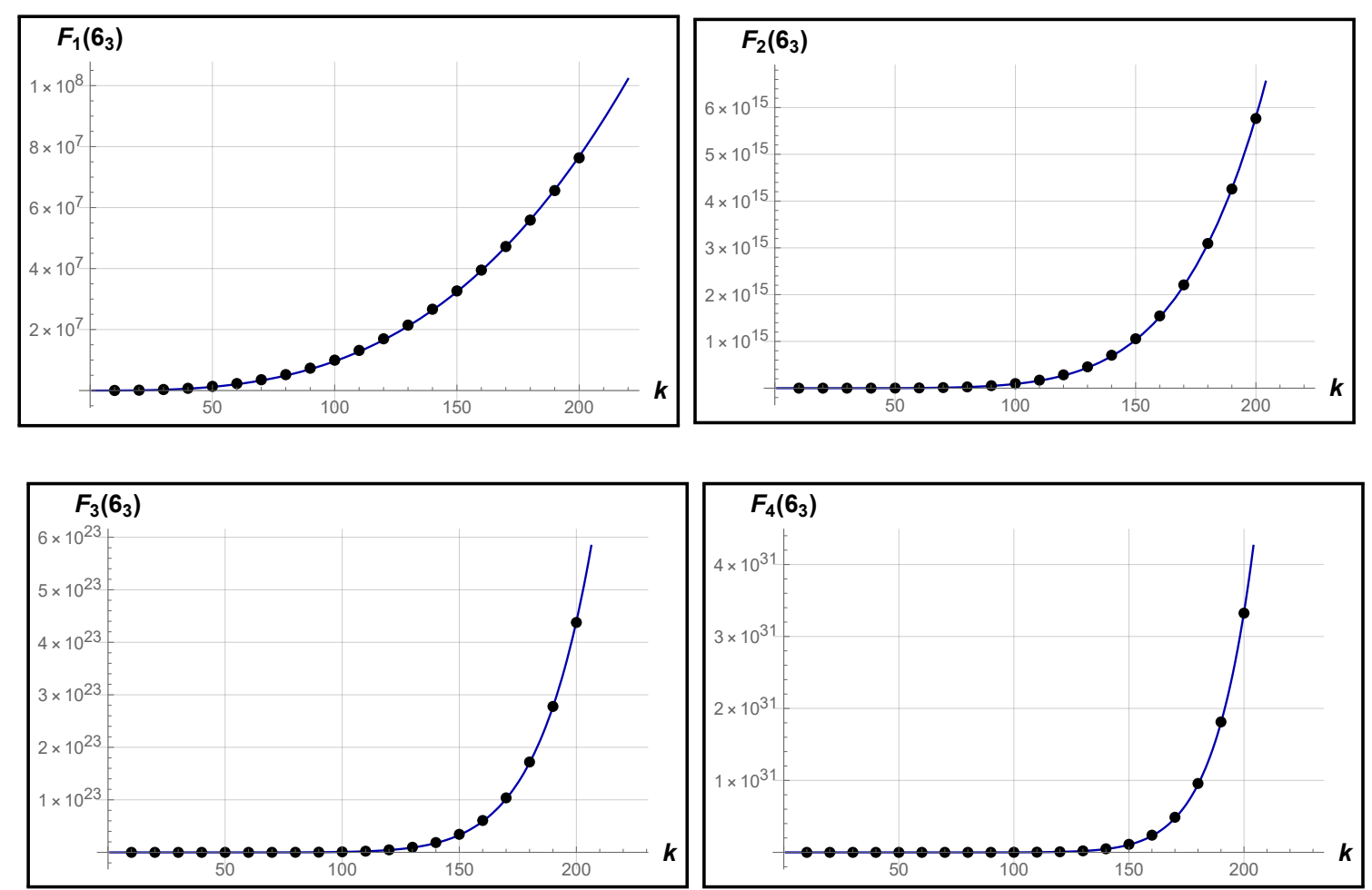

Figure 23. The variation of the function $F_{m}$ with $k$ for $6_{3}$ knot. The solid line denotes the function $C_{m} k^{3 m}$ with values of $C_{m}$ given in table 14 . The $\bullet$ indicates the numerical values of $F_{m}$ obtained from explicit computations.

\begin{tabular}{|c|c|}
\hline$m$ & $\lim _{k \rightarrow \infty} \mathcal{R}_{m}$ \\
\hline 2 & $0.0169586610 \pm 0.0042599254$ \\
3 & $0.0144352100 \pm 0.0030269172$ \\
4 & $0.0135581550 \pm 0.0026399898$ \\
5 & $0.0130967624 \pm 0.0024536120$ \\
6 & $0.0128029098 \pm 0.0023446021$ \\
7 & $0.0125940093 \pm 0.0022732852$ \\
8 & $0.0124347393 \pm 0.0022230911$ \\
9 & $0.0123074665 \pm 0.0021858981$ \\
10 & $0.0122023867 \pm 0.0021572646$ \\
\hline
\end{tabular}

Table 15. The $k \rightarrow \infty$ values of the Rényi entropies associated with the state $\left|6_{3} \# T_{2,2}\right\rangle$.

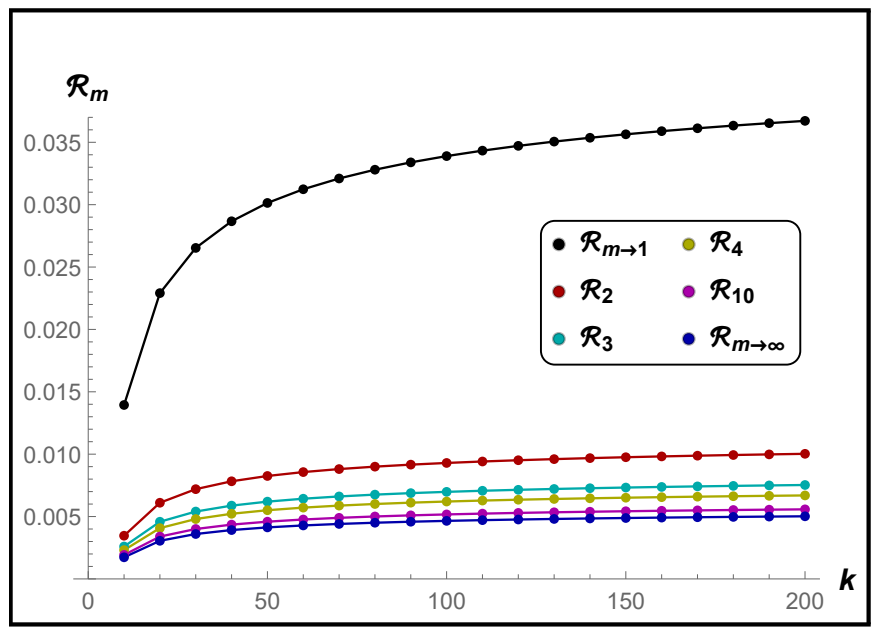

Figure 24. Variation of Rényi entropies for the state $\left|6_{3} \# T_{2,2}\right\rangle$ with $k$. 
The partial tracing of one of the torus boundary gives the reduced density matrix $\sigma\left(\mathcal{K} \# T_{2,2}\right)(3.8)$, which provides the starting point for computing various entanglement measures. The Rényi entropy with index $m$ can be obtained from the $m$-moment or the trace of the $m^{\text {th }}$ power of the matrix $\sigma\left(\mathcal{K} \# T_{2,2}\right)$ using (2.16). Incidentally, the trace operation on the reduced density matrix can be viewed as gluing the two oppositely oriented torus boundaries resulting in a closed three-manifold $M_{\mathcal{K}}$ giving the Chern-Simons partition function $Z\left(M_{\mathcal{K}}\right)=\operatorname{Tr}\left[\sigma\left(\mathcal{K} \# T_{2,2}\right)\right]$. The subscript on the three-manifold $M_{\mathcal{K}}$ keeps track of the knot involved in the two-component link $\mathcal{L}=\mathcal{K} \# T_{2,2}$. We would like to mention that the authors of the ref. [24] study the perturbative expansion of the $\operatorname{SL}(2, \mathbb{C})$ Chern-Simons partition function of closed three-manifolds which are obtained from the Dehn filling of link complements. It would be worthwhile to explore the results of [24] in the set-up of our paper. In particular, it will be interesting to see if the manifold $M_{\mathcal{K}}$ can be viewed as the Dehn filling of certain link complement which can enable us to obtain the subleading terms in the expansion of $Z\left(M_{\mathcal{K}}\right)$. We leave this discussion for future work.

We were able to reproduce similar geometrical results for the higher moments of the reduced density matrix. Our crucial observation that the replica trick in the entanglement context is quantified by the connected sums of knots led to (3.14) and (3.15) respectively. Thus we have shown that all the $m$-moments of the matrix $\sigma\left(\mathcal{K} \# T_{2,2}\right)$ are the Chern-Simons partition functions $Z\left(M_{\mathcal{K}_{m}}\right)$ where $\mathcal{K}_{m}=\mathcal{K} \# \mathcal{K} \ldots \# \mathcal{K}$ denotes the connected sum of $m$ copies of $\mathcal{K}$. As a result, the Rényi entropies with index $m$ can be written in terms of the three-manifold invariants $Z\left(M_{\mathcal{K}_{m}}\right)$ as given in (3.16).

Even though we cannot visualise the explicit topology of $M_{\mathcal{K}}$, we were able to address the large $k$ asymptotics of $Z\left(M_{\mathcal{K}}\right)$ for $\mathrm{SU}(2)$ and $\mathrm{SO}(3)$ gauge groups. Using the asymptotics of Turaev-Viro invariant of $S^{3} \backslash \mathcal{K}$ evaluated at $e^{\frac{\pi i}{k+2}}$, we were able to show in (3.24), that $\operatorname{Tr}\left[\sigma\left(\mathcal{K} \# T_{2,2}\right)\right]=Z\left(M_{\mathcal{K}}\right)$ for $\mathrm{SU}(2)$ group can grow at most polynomially in $k$. In contrast, the traces show an exponential growth in $k$ for the $\mathrm{SO}(3)$ group. Incorporating the well known Kashaev's conjecture [18], we conjecture that the leading term of $\ln \operatorname{Tr}\left[\sigma\left(\mathcal{K} \# T_{2,2}\right)\right]$ and hence $\ln Z\left(M_{\mathcal{K}}\right)$ for $\mathrm{SO}(3)$ gauge group, captures the hyperbolic volume of the knot complement $S^{3} \backslash \mathcal{K}$ in the large $k$ limit. These are given as conjecture (3.33) and corollary (3.39) respectively. We have performed numerical computations for torus and non-torus knots up to six crossings to validate our conjecture and proposal.

We further propose an asymptotic form for $\operatorname{Tr}\left[\sigma^{m}\left(\mathcal{K} \# T_{2,2}\right)\right]=Z\left(M_{\mathcal{K}_{m}}\right)$ for $\mathrm{SO}(3)$ gauge group, in the large $k$ limit (3.50). Using such a form, we see that the Rényi entropies converge to a finite number as $k \rightarrow \infty$ (3.51). We would like to mention that currently we do not have an understanding of the geometric or topological interpretation of the large $k$ limiting values of Rényi entropies, and it will be worthwhile to explore this aspect in the context of $\mathrm{SO}(3)$ Chern-Simons theories.

\section{Acknowledgments}

SD is supported by the NSFC grants No. 12050410249 and No. 11975158. PR would like to thank SERB (MATRICS) MTR/2019/000956 funding. VKS would like to thank Rama Mishra for helpful discussion and acknowledge the hospitality of department of Mathematics, IISER, Pune (India) where this work was done during his visit as visiting fellow. BPM acknowledges the Research Grant for Faculty under IoE Scheme (number 6031). 


\section{A $\quad \mathrm{SO}(3)$ Chern-Simons theory and hyperbolic volumes of $S^{3} \backslash \mathcal{K}$}

In the appendix, we will verify the following conjecture for some of the prime knots:

$$
\lim _{k \rightarrow \infty} \frac{\ln \left|J_{k}\left(\mathcal{K} ; e^{\frac{4 \pi i}{k+1}}\right)\right|}{k}=\frac{\operatorname{Vol}\left(S^{3} \backslash \mathcal{K}\right)}{4 \pi} .
$$

For this, we use the equality between the colored Jones and Kashaev's invariant. In particular, setting $n=(k+1)$ with $k \in 2 \mathbb{Z}$, we have:

$$
\left|\operatorname{Kas}_{n}\left(\mathcal{K} ; q=e^{\frac{4 \pi i}{n}}\right)\right|=\left|J_{n-1}\left(\mathcal{K} ; q=e^{\frac{4 \pi i}{n}}\right)\right| .
$$

This has already been discussed in section 3 around eq. (3.36). So, we study the asymptotics of the above Kashaev's invariant for prime knots up to six crossings using the stationary phase approximation prescribed in $[18,22]$. This approach works for those knots whose invariant can be written in terms of $q$-Pochhammer symbols

$$
(q)_{\ell}=\prod_{j=1}^{\ell}\left(1-q^{j}\right) .
$$

We consider the limit

$$
q=\exp \left(\frac{4 \pi i}{n}\right), \quad n \rightarrow \infty .
$$

The aim is to obtain the maximum summand that dominates the asymptotic of the Kashaev's invariant in the above limit. For this, the summations appearing in the Kashaev's invariant are converted into integral expressions of the following form:

$$
\operatorname{Kas}_{n}\left(\mathcal{K} ; q=e^{\frac{4 \pi i}{n}}\right) \sim \iint \ldots \int d x_{1} d x_{2} \ldots d x_{p} \exp \left(\frac{n}{4 \pi i} V_{\mathcal{K}}\left(x_{1}, \ldots, x_{p}\right)\right)
$$

where the integration variable $x_{i}=q^{a_{i}}$ with $a_{i}$ being the summation variable in the Kashaev's invariant. The potential $V_{\mathcal{K}}$ is formally obtained by replacing each of the $q$-Pochhammer symbols appearing in the Kashaev's invariant by their asymptotic expressions. This is done by approximating the $q$-Pochhammer by a dilogarithm function as shown below:

$$
\begin{aligned}
\ln (q)_{\ell} & =\sum_{j=1}^{\ell} \ln \left(1-e^{\frac{4 \pi i j}{n}}\right) \sim \frac{n}{4 \pi i} \int_{0}^{\frac{4 \pi i \ell}{n}} \ln \left(1-e^{t}\right) d t \\
\Longrightarrow(q)_{\ell} & \sim \exp \left[\frac{n}{4 \pi i}\left(\frac{\pi^{2}}{6}-\operatorname{Li}_{2}\left(q^{\ell}\right)\right)\right] .
\end{aligned}
$$

Once the potential $V_{\mathcal{K}}\left(x_{1}, \ldots, x_{p}\right)$ is known, we can apply the stationary phase approximation for the integral (A.5) in the large $n$ limit, and obtain a saddle point solution $\left(x_{1}^{0}, \ldots, x_{p}^{0}\right)$ which satisfies:

$$
\left.\frac{\partial V_{\mathcal{K}}\left(x_{1}, \ldots, x_{p}\right)}{\partial x_{j}}\right|_{\left(x_{1}, \ldots, x_{p}\right)=\left(x_{1}^{0}, \ldots, x_{p}^{0}\right)}=0, \quad \forall j=1,2, \ldots, p
$$


As a result, we can write the following limit:

$$
\lim _{n \rightarrow \infty} \frac{4 \pi}{n} \ln \operatorname{Kas}_{n}\left(\mathcal{K} ; e^{\frac{4 \pi i}{n}}\right)=-i V_{\mathcal{K}}\left(x_{1}^{0}, \ldots, x_{p}^{0}\right) .
$$

The real part of the right hand side or the imaginary part of the potential is expected to coincide with the hyperbolic volume of $S^{3} \backslash \mathcal{K}$ :

$$
\operatorname{Re}\left[-i V_{\mathcal{K}}\left(x_{1}^{0}, \ldots, x_{p}^{0}\right)\right]=\operatorname{Im}\left[V_{\mathcal{K}}\left(x_{1}^{0}, \ldots, x_{p}^{0}\right)\right]=\operatorname{Vol}\left(S^{3} \backslash \mathcal{K}\right) .
$$

Thus, finally we can arrive at

$$
\lim _{n \rightarrow \infty} \frac{\ln \left|\operatorname{Kas}_{n}\left(\mathcal{K} ; e^{\frac{4 \pi i}{n}}\right)\right|}{n}=\lim _{n \rightarrow \infty} \frac{\ln \left|J_{n-1}\left(\mathcal{K} ; e^{\frac{4 \pi i}{n}}\right)\right|}{n}=\frac{\operatorname{Vol}\left(S^{3} \backslash \mathcal{K}\right)}{4 \pi} .
$$

In the following, we will verify (A.9) and (A.10) for prime knots up to six crossings.

- $\boldsymbol{S}^{\mathbf{3}} \backslash \mathbf{3}_{\mathbf{1}}$. The colored Jones polynomial associated with $3_{1}$ knot colored by an SU(2) irrep with Dynkin label $\alpha$ is given as:

$$
J_{\alpha}\left(3_{1}, q\right)=\sum_{j=-\alpha / 2}^{\alpha / 2}\left(\frac{q^{-\frac{3 \alpha^{2}}{2}-\frac{5 \alpha}{2}+6 j^{2}-5 j+1}-q^{-\frac{3 \alpha^{2}}{2}-\frac{5 \alpha}{2}+6 j^{2}+j}}{q^{\alpha+1}-1}\right) .
$$

The Kashaev's invariant for the $3_{1}$ knot is given as,

$$
\operatorname{Kas}_{n}\left(3_{1} ; q\right)=\sum_{j=0}^{n-1}(q)_{j}
$$

We have numerically verified that:

$$
\left|J_{n-1}\left(3_{1}, e^{\frac{4 \pi i}{n}}\right)\right|=\left|\operatorname{Kas}_{n}\left(3_{1} ; e^{\frac{4 \pi i}{n}}\right)\right|, \quad n=1,3,5,7, \ldots
$$

Using (A.6) and setting $x=q^{j}$ with $q=e^{\frac{4 \pi i}{n}}$, we will have the following expression in large $n$ limit:

$$
\operatorname{Kas}_{n}\left(3_{1} ; e^{\frac{4 \pi i}{n}}\right) \sim \int d x \exp \left(\frac{n}{4 \pi i} V_{3_{1}}(x)\right),
$$

where the potential is given as

$$
V_{3_{1}}(x)=\frac{\pi^{2}}{6}-\operatorname{Li}_{2}(x)
$$

The saddle point is $x^{0}=0$ and corresponding potential is given as:

$$
V_{3_{1}}\left(x^{0}\right)=\frac{\pi^{2}}{6} \quad \Longrightarrow \quad \operatorname{Im}\left[V_{3_{1}}\left(x^{0}\right)\right]=0 .
$$

This gives the vanishing hyperbolic volume of $S^{3} \backslash 3_{1}$ which is expected since $3_{1}$ is a torus knot. This verifies (A.10). 
- $\boldsymbol{S}^{\mathbf{3}} \backslash \mathbf{4}_{\mathbf{1}}$. The Jones polynomial associated with $4_{1}$ knot colored by an $\mathrm{SU}(2)$ irrep with Dynkin label $\alpha$ is given as:

$$
J_{\alpha}\left(4_{1}, q\right)=\sum_{a=0}^{\alpha} \prod_{j=1}^{a}\left(q^{\frac{1}{2}(\alpha-j+1)}-q^{-\frac{1}{2}(\alpha-j+1)}\right)\left(q^{\frac{1}{2}(\alpha+j+1)}-q^{-\frac{1}{2}(\alpha+j+1)}\right)
$$

The Kashaev's invariant for the $4_{1}$ knot is given as [18],

$$
\operatorname{Kas}_{n}\left(4_{1} ; q\right)=\sum_{j=0}^{n-1}\left|(q)_{j}\right|^{2}
$$

We have numerically verified that:

$$
\left|J_{n-1}\left(4_{1}, e^{\frac{4 \pi i}{n}}\right)\right|=\left|\operatorname{Kas}_{n}\left(4_{1} ; e^{\frac{4 \pi i}{n}}\right)\right|, \quad n=1,3,5,7, \ldots .
$$

Defining $x=q^{j}$ with $q=e^{\frac{4 \pi i}{n}}$, and using (A.6), we will have the following expression in the large $n$ limit:

$$
\operatorname{Kas}_{n}\left(4_{1} ; e^{\frac{4 \pi i}{n}}\right) \sim \int d x \exp \left(\frac{n}{4 \pi i} V_{4_{1}}(x)\right),
$$

where the potential is given as [18]:

$$
V_{4_{1}}(x)=\mathrm{Li}_{2}\left(x^{-1}\right)-\mathrm{Li}_{2}(x) .
$$

The saddle point is $x^{0}=e^{-\frac{i \pi}{3}}$ at which we have:

$$
\operatorname{Im}\left[V_{4_{1}}\left(x^{0}\right)\right] \approx 2.0298832128193072500424 \ldots
$$

This coincides with the hyperbolic volume of $S^{3} \backslash 4_{1}$ and verifies (A.10).

- $\boldsymbol{S}^{\mathbf{3}} \backslash \mathbf{5}_{\mathbf{1}}$. The colored Jones polynomial associated with $5_{1}$ knot colored by an SU(2) irrep with Dynkin label $\alpha$ is given as:

$$
J_{\alpha}\left(5_{1}, q\right)=\sum_{j=-\alpha / 2}^{\alpha / 2}\left(\frac{q^{-\frac{5 \alpha^{2}}{2}-\frac{9 \alpha}{2}+10 j^{2}-7 j+1}-q^{-\frac{5 \alpha^{2}}{2}-\frac{9 \alpha}{2}+10 j^{2}+3 j}}{q^{\alpha+1}-1}\right) .
$$

The Kashaev's invariant for the $5_{1}$ knot is given as,

$$
\operatorname{Kas}_{n}\left(5_{1} ; q\right)=\sum_{\substack{j_{1}, j_{2}=0 \\ 0 \leq j_{1}+j_{2} \leq n-1}}^{n-1} q^{-j_{1} j_{2}}(q)_{j_{1}+j_{2}}
$$

We have numerically verified that:

$$
\left|J_{n-1}\left(5_{1}, e^{\frac{4 \pi i}{n}}\right)\right|=\left|\operatorname{Kas}_{n}\left(5_{1} ; e^{\frac{4 \pi i}{n}}\right)\right|, \quad n=1,3,5,7, \ldots .
$$

Defining $x_{1}=q^{j_{1}}$ and $x_{2}=q^{j_{2}}$ with $q=e^{\frac{4 \pi i}{n}}$, using (A.6) and noting that

$$
q^{-j_{1} j_{2}}=e^{-\frac{4 \pi i j_{1} j_{2}}{n}} \sim e^{-\frac{n}{4 \pi i}\left(\ln x_{1}\right)\left(\ln x_{2}\right)},
$$


we will have the following expression in the large $n$ limit:

$$
\operatorname{Kas}_{n}\left(5_{1} ; e^{\frac{4 \pi i}{n}}\right) \sim \iint d x_{1} d x_{2} \exp \left(\frac{n}{4 \pi i} V_{5_{1}}\left(x_{1}, x_{2}\right)\right),
$$

where the potential is given as

$$
V_{5_{1}}\left(x_{1}, x_{2}\right)=\frac{\pi^{2}}{6}-\operatorname{Li}_{2}\left(x_{1} x_{2}\right)-\left(\ln x_{1}\right)\left(\ln x_{2}\right) .
$$

The saddle points are given as:

$$
\left(x_{1}^{0}, x_{2}^{0}\right)=\left(-\frac{\sqrt{5}+1}{2},-\frac{\sqrt{5}+1}{2}\right),\left(\frac{\sqrt{5}-1}{2}, \frac{\sqrt{5}-1}{2}\right) .
$$

At both of these saddle points, we find that $\operatorname{Im}\left[V_{5_{1}}\left(x_{1}^{0}, x_{2}^{0}\right)\right]=0$. This gives the vanishing hyperbolic volume of $S^{3} \backslash 5_{1}$ which is expected since $5_{1}$ is a torus knot. This verifies (A.10).

- $\boldsymbol{S}^{\mathbf{3}} \backslash \mathbf{5}_{\mathbf{2}}$. The Jones polynomial associated with $5_{2}$ knot colored by an SU(2) irrep with Dynkin label $\alpha$ is given as:

$$
J_{\alpha}\left(5_{2}, q\right)=\sum_{a_{1}=0}^{\alpha} \sum_{a_{2}=0}^{a_{1}} \frac{(-1)^{a_{2}} q^{a_{1}+\frac{1}{2} a_{2}\left(5 a_{2}+3\right)}\left(1-q^{2 a_{2}+1}\right)\left(\begin{array}{c}
a_{1} \\
a_{2}
\end{array}\right)_{q}\left(q^{-\alpha} ; q\right)_{a_{1}}\left(q^{\alpha+2} ; q\right)_{a_{1}}}{\left(q^{a_{2}+1} ; q\right)_{a_{1}+1}},
$$

where the following definitions are used:

$$
(z ; q)_{\ell}=\prod_{j=0}^{\ell-1}\left(1-z q^{j}\right), \quad\left(\begin{array}{l}
x \\
y
\end{array}\right)_{q}=\frac{(q ; q)_{x}}{(q ; q)_{y}(q ; q)_{x-y}} .
$$

The Kashaev's invariant for the $5_{2}$ knot is given as [18],

$$
\operatorname{Kas}_{n}\left(5_{2} ; q\right)=\sum_{j_{1}=0}^{n-1} \sum_{j_{2}=0}^{j_{1}} q^{-\left(j_{1}+1\right) j_{2}} \frac{(q)_{j_{1}}(q)_{j_{1}}}{(q)_{j_{2}}^{*}}
$$

We have numerically checked that

$$
\left|J_{n-1}\left(5_{2}, e^{\frac{4 \pi i}{n}}\right)\right|=\left|\operatorname{Kas}_{n}\left(5_{2} ; e^{\frac{4 \pi i}{n}}\right)\right|, \quad n=1,3,5,7, \ldots .
$$

Setting $x_{1}=q^{j_{1}}$ and $x_{2}=q^{j_{2}}$ with $q=e^{\frac{4 \pi i}{n}}$, using (A.6) and (A.26), we have the following asymptotic for the Kashaev's invariant in the large $n$ limit:

$$
\operatorname{Kas}_{n}\left(5_{2} ; e^{\frac{4 \pi i}{n}}\right) \sim \iint d x_{1} d x_{2} \exp \left(\frac{n}{4 \pi i} V_{5_{2}}\left(x_{1}, x_{2}\right)\right),
$$

where the potential is given as [18]:

$$
V_{5_{2}}\left(x_{1}, x_{2}\right)=\frac{\pi^{2}}{2}-2 \operatorname{Li}_{2}\left(x_{1}\right)-\operatorname{Li}_{2}\left(x_{2}^{-1}\right)-\left(\ln x_{1}\right)\left(\ln x_{2}\right) .
$$

The saddle point is approximately given as:

$$
\left(\begin{array}{l}
x_{1}^{0} \\
x_{2}^{0}
\end{array}\right)=\left(\begin{array}{l}
0.3376410213776269870195456-0.5622795120623012438991821 i \\
0.1225611668766536199752455+0.7448617666197442365931704 i
\end{array}\right)
$$

at which the imaginary part of the potential is given as:

$$
\operatorname{Im}\left[V_{5_{2}}\left(x_{1}^{0}, x_{2}^{0}\right)\right] \approx 2.82812208833078316276390 \ldots
$$

This coincides with the hyperbolic volume of $S^{3} \backslash 5_{2}$ and verifies (A.10). 
- $\boldsymbol{S}^{\mathbf{3}} \backslash \mathbf{6}_{\mathbf{1}}$. The Jones polynomial associated with $6_{1}$ knot colored by an $\mathrm{SU}(2)$ irrep with Dynkin label $\alpha$ is given as:

$$
J_{\alpha}\left(6_{1}, q\right)=\sum_{a_{1}=0}^{\alpha} \sum_{a_{2}=0}^{a_{1}}\left(q^{-\alpha a_{1}+a_{2}^{2}+a_{2}-a_{1}} \frac{(q ; q)_{a_{1}}(q ; q)_{\alpha}\left(q^{\alpha+2} ; q\right)_{a_{1}}}{(q ; q)_{a_{1}-a_{2}}(q ; q)_{a_{2}}(q ; q)_{\alpha-a_{1}}}\right) .
$$

The Kashaev's invariant for the $6_{1}$ knot is given as [18],

$$
\operatorname{Kas}_{n}\left(6_{1} ; q\right)=\sum_{\substack{j_{1}, j_{2}, j_{3}=0 \\ j_{1}+j_{2} \leq j_{3}}}^{n-1} q^{\left(j_{3}-j_{1}-j_{2}\right)\left(j_{3}-j_{1}+1\right)} \frac{\left|(q)_{j_{3}}\right|^{2}}{(q)_{j_{1}}(q)_{j_{2}}^{*}}
$$

We have numerically checked that

$$
\left|J_{n-1}\left(6_{1}, e^{\frac{4 \pi i}{n}}\right)\right|=\left|\operatorname{Kas}_{n}\left(6_{1} ; e^{\frac{4 \pi i}{n}}\right)\right|, \quad n=1,3,5,7, \ldots .
$$

Setting $x_{1}=q^{j_{1}}, x_{2}=q^{j_{2}}, x_{3}=q^{j_{3}}$ and using (A.6), we have the following asymptotic for the Kashaev's invariant in the large $n$ limit:

$$
\operatorname{Kas}_{n}\left(6_{1} ; e^{\frac{4 \pi i}{n}}\right) \sim \iiint d x_{1} d x_{2} d x_{3} \exp \left(\frac{n}{4 \pi i} V_{6_{1}}\left(x_{1}, x_{2}, x_{3}\right)\right),
$$

where the potential is given as [18]:

$$
V_{6}\left(x_{1}, x_{2}, x_{3}\right)=\mathrm{Li}_{2}\left(x_{1}\right)-\mathrm{Li}_{2}\left(x_{2}^{-1}\right)+\mathrm{Li}_{2}\left(x_{3}^{-1}\right)-\mathrm{Li}_{2}\left(x_{3}\right)+\ln \left(\frac{x_{1}}{x_{3}}\right) \ln \left(\frac{x_{1} x_{2}}{x_{3}}\right)-2 \pi i \ln \left(\frac{x_{1}}{x_{3}}\right) .
$$

The saddle point is approximately given as:

$$
\left(\begin{array}{l}
x_{1}^{0} \\
x_{2}^{0} \\
x_{3}^{0}
\end{array}\right)=\left(\begin{array}{l}
0.17384979383679558571428+1.06907189987572611850590 i \\
0.32204184201246211276846+0.15777973787085281762619 i \\
0.27872641157714581676289-0.48341992018615350391557 i
\end{array}\right)
$$

at which the imaginary part of the potential is given as:

$$
\operatorname{Im}\left[V_{6_{1}}\left(x_{1}^{0}, x_{2}^{0}\right)\right] \approx 3.163963228883143983991 \ldots .
$$

This coincides with the hyperbolic volume of $S^{3} \backslash 6_{1}$ and verifies (A.10).

- $\boldsymbol{S}^{\mathbf{3}} \backslash \mathbf{6}_{\mathbf{2}}$. The Jones polynomial associated with $6_{2}$ knot colored by an SU(2) irrep with Dynkin label $\alpha$ is given as:

$$
\begin{gathered}
J_{\alpha}\left(6_{2}, q\right)=\sum_{a_{1}=0}^{\alpha} \sum_{a_{2}=0}^{a_{1}} \sum_{a_{3}=0}^{a_{2}}\left((-1)^{a_{2}+a_{3}} q^{\frac{1}{2}\left(-2 \alpha a_{1}-a_{2}^{2}+2 a_{1} a_{2}+a_{2}+a_{3}^{2}+a_{3}\right)}\left(\begin{array}{c}
a_{1} \\
a_{2}
\end{array}\right)_{q}\left(\begin{array}{c}
a_{2} \\
a_{3}
\end{array}\right)_{q}(q ; q)_{a_{2}}\right. \\
\left.\left(q^{a_{3}+1} ; q\right)_{a_{1}-a_{2}}\left(\begin{array}{c}
\alpha \\
a_{1}
\end{array}\right)_{q}\left(q^{\alpha+2} ; q\right)_{a_{1}}\right) .
\end{gathered}
$$


The Kashaev's invariant for the $6_{2}$ knot is given as [22],

$$
\operatorname{Kas}_{n}\left(6_{2} ; q\right)=\sum_{\substack{j_{1}, j_{2}, j_{3}=0 \\ j_{1} \leq j_{2} \\ 0 \leq j_{1}+j_{3} \leq n-1}}^{n-1} q^{-j_{1}\left(j_{2}+j_{3}+1\right)} \frac{(q)_{j_{2}}(q)_{j_{2}}(q)_{j_{1}+j_{3}}}{\left|(q)_{j_{1}}\right|^{2}(q)_{j_{2}-j_{1}}}
$$

We have numerically checked that

$$
\left|J_{n-1}\left(6_{2}, e^{\frac{4 \pi i}{n}}\right)\right|=\left|\operatorname{Kas}_{n}\left(6_{2} ; e^{\frac{4 \pi i}{n}}\right)\right|, \quad n=1,3,5,7, \ldots .
$$

Setting $x_{1}=q^{j_{1}}, x_{2}=q^{j_{2}}, x_{3}=q^{j_{3}}$ and using (A.6), we have the following asymptotic for the Kashaev's invariant in the large $n$ limit:

$$
\operatorname{Kas}_{n}\left(6_{2} ; e^{\frac{4 \pi i}{n}}\right) \sim \iiint d x_{1} d x_{2} d x_{3} \exp \left(\frac{n}{4 \pi i} V_{6_{2}}\left(x_{1}, x_{2}, x_{3}\right)\right),
$$

where the potential is given as [22]:

$$
V_{6_{2}}\left(x_{1}, x_{2}, x_{3}\right)=\mathrm{Li}_{2}\left(x_{1}\right)-\mathrm{Li}_{2}\left(x_{1}^{-1}\right)+\operatorname{Li}_{2}\left(x_{1}^{-1} x_{2}\right)-2 \operatorname{Li}_{2}\left(x_{2}\right)-\operatorname{Li}_{2}\left(x_{1} x_{3}\right)-\left(\ln x_{1}\right)\left(\ln x_{2} x_{3}\right)+\frac{\pi^{2}}{3} .
$$

The saddle point is approximately given as:

$$
\left(\begin{array}{l}
x_{1}^{0} \\
x_{2}^{0} \\
x_{3}^{0}
\end{array}\right)=\left(\begin{array}{c}
0.0904326688828523150727+1.6028830694139448442811 i \\
-0.23270516544550685831671-1.09381177109577462363025 i \\
-0.96491338528654243537970-0.62189628352233029773000 i
\end{array}\right)
$$

at which the imaginary part of the potential is given as:

$$
\operatorname{Im}\left[V_{6_{2}}\left(x_{1}^{0}, x_{2}^{0}\right)\right] \approx 4.400832516123046101441 \ldots .
$$

This coincides with the hyperbolic volume of $S^{3} \backslash 6_{2}$ and verifies (A.10).

- $\boldsymbol{S}^{\mathbf{3}} \backslash \mathbf{6}_{\mathbf{3}}$. The Jones polynomial associated with $6_{3}$ knot colored by an SU(2) irrep with Dynkin label $\alpha$ is given as:

$$
\begin{gathered}
J_{\alpha}\left(6_{3}, q\right)=\sum_{a_{1}=0}^{\alpha} \sum_{a_{2}=0}^{a_{1}} \sum_{a_{3}=0}^{a_{2}}\left((-1)^{a_{1}+a_{3}} q^{\frac{1}{2}\left(-a_{1}\left(2 \alpha+2 a_{2}+1\right)+a_{1}^{2}+a_{3}^{2}-2 a_{2}+a_{3}\right)}\left(\begin{array}{c}
a_{1} \\
a_{2}
\end{array}\right)_{q}\left(\begin{array}{c}
a_{2} \\
a_{3}
\end{array}\right)_{q}(q ; q)_{a_{2}}\right. \\
\left.\left(q^{a_{3}+1} ; q\right)_{a_{1}-a_{2}}\left(\begin{array}{c}
\alpha \\
a_{1}
\end{array}\right)_{q}\left(q^{\alpha+2} ; q\right)_{a_{1}}\right) .
\end{gathered}
$$

The Kashaev's invariant for the $6_{3}$ knot is given as [22],

$$
\operatorname{Kas}_{n}\left(6_{3} ; q\right)=\sum_{\substack{j_{1}, j_{2}, j_{3}=0 \\ 0 \leq j_{1}+j_{2}+j_{3} \leq n-1}}^{n-1} q^{\left(j_{1}+1\right)\left(j_{2}-j_{3}\right)} \frac{\left|(q)_{j_{1}+j_{2}+j_{3}}\right|^{2}(q)_{j_{1}+j_{2}}^{*}(q)_{j_{1}+j_{3}}}{\left|(q)_{j_{2}}\right|^{2}\left|(q)_{j_{3}}\right|^{2}}
$$


We have numerically checked that

$$
\left|J_{n-1}\left(6_{3}, e^{\frac{4 \pi i}{n}}\right)\right|=\left|\operatorname{Kas}_{n}\left(6_{3} ; e^{\frac{4 \pi i}{n}}\right)\right|, \quad n=1,3,5,7, \ldots .
$$

Setting $x_{1}=q^{j_{1}}, x_{2}=q^{j_{2}}, x_{3}=q^{j_{3}}$ and using (A.6), we have the following asymptotic for the Kashaev's invariant in the large $n$ limit:

$$
\operatorname{Kas}_{n}\left(6_{3} ; e^{\frac{4 \pi i}{n}}\right) \sim \iiint d x_{1} d x_{2} d x_{3} \exp \left(\frac{n}{4 \pi i} V_{6_{3}}\left(x_{1}, x_{2}, x_{3}\right)\right),
$$

where the potential is given as [22]:

$$
\begin{aligned}
V_{63}\left(x_{1}, x_{2}, x_{3}\right)= & \operatorname{Li}_{2}\left(x_{2}\right)-\mathrm{Li}_{2}\left(x_{2}^{-1}\right)+\operatorname{Li}_{2}\left(x_{3}\right)-\operatorname{Li}_{2}\left(x_{3}^{-1}\right)+\operatorname{Li}_{2}\left(x_{1}^{-1} x_{2}^{-1}\right)-\operatorname{Li}_{2}\left(x_{1} x_{3}\right) \\
& -\operatorname{Li}_{2}\left(x_{1} x_{2} x_{3}\right)+\operatorname{Li}_{2}\left(x_{1}^{-1} x_{2}^{-1} x_{3}^{-1}\right)+\left(\ln x_{1}\right) \ln \left(x_{2} / x_{3}\right) .
\end{aligned}
$$

The saddle point is approximately given as:

$$
\left(\begin{array}{c}
x_{1}^{0} \\
x_{2}^{0} \\
x_{3}^{0}
\end{array}\right)=\left(\begin{array}{c}
0.20432287067507999865586-0.97890355220475859397659 i \\
1.6083782859756256503564+0.5587518814119368864517 i \\
0.55478836677297306027131+0.19273391491468578161798 i
\end{array}\right)
$$

at which the imaginary part of the potential is given as:

$$
\operatorname{Im}\left[V_{6_{3}}\left(x_{1}^{0}, x_{2}^{0}\right)\right] \approx 5.693021091281300765112 \ldots
$$

This coincides with the hyperbolic volume of $S^{3} \backslash 6_{3}$ and verifies (A.10).

Open Access. This article is distributed under the terms of the Creative Commons Attribution License (CC-BY 4.0), which permits any use, distribution and reproduction in any medium, provided the original author(s) and source are credited.

\section{References}

[1] E. Witten, Quantum Field Theory and the Jones Polynomial, Commun. Math. Phys. 121 (1989) 351 [INSPIRE].

[2] A. Kitaev and J. Preskill, Topological entanglement entropy, Phys. Rev. Lett. 96 (2006) 110404 [hep-th/0510092] [INSPIRE].

[3] M. Levin and X.-G. Wen, Detecting Topological Order in a Ground State Wave Function, Phys. Rev. Lett. 96 (2006) 110405 [cond-mat/0510613] [INSPIRE].

[4] S. Dong, E. Fradkin, R.G. Leigh and S. Nowling, Topological Entanglement Entropy in Chern-Simons Theories and Quantum Hall Fluids, JHEP 05 (2008) 016 [arXiv:0802.3231] [INSPIRE].

[5] V. Balasubramanian, J.R. Fliss, R.G. Leigh and O. Parrikar, Multi-Boundary Entanglement in Chern-Simons Theory and Link Invariants, JHEP 04 (2017) 061 [arXiv:1611.05460] [INSPIRE].

[6] S. Dwivedi, V.K. Singh, S. Dhara, P. Ramadevi, Y. Zhou and L.K. Joshi, Entanglement on linked boundaries in Chern-Simons theory with generic gauge groups, JHEP 02 (2018) 163 [arXiv: 1711.06474] [INSPIRE]. 
[7] V. Balasubramanian, M. DeCross, J. Fliss, A. Kar, R.G. Leigh and O. Parrikar, Entanglement Entropy and the Colored Jones Polynomial, JHEP 05 (2018) 038 [arXiv:1801.01131] [INSPIRE].

[8] L.-Y. Hung, Y.-S. Wu and Y. Zhou, Linking Entanglement and Discrete Anomaly, JHEP 05 (2018) 008 [arXiv: 1801.04538] [inSPIRE].

[9] D. Melnikov, A. Mironov, S. Mironov, A. Morozov and A. Morozov, From Topological to Quantum Entanglement, JHEP 05 (2019) 116 [arXiv:1809.04574] [INSPIRE].

[10] G. Camilo, D. Melnikov, F. Novaes and A. Prudenziati, Circuit Complexity of Knot States in Chern-Simons theory, JHEP 07 (2019) 163 [arXiv: 1903.10609] [InSPIRE].

[11] S. Dwivedi, V.K. Singh, P. Ramadevi, Y. Zhou and S. Dhara, Entanglement on multiple $S^{2}$ boundaries in Chern-Simons theory, JHEP 08 (2019) 034 [arXiv: 1906.11489] [INSPIRE].

[12] M. Buican and R. Radhakrishnan, Galois conjugation and multiboundary entanglement entropy, JHEP 12 (2020) 045 [arXiv: 1912.04937] [INSPIRE].

[13] Y. Zhou, 3d One-form Mixed Anomaly and Entanglement Entropy, JHEP 07 (2019) 091 [arXiv: 1904.06924] [INSPIRE].

[14] S. Dwivedi, A. Addazi, Y. Zhou and P. Sharma, Multi-boundary entanglement in Chern-Simons theory with finite gauge groups, JHEP 04 (2020) 158 [arXiv: 2003.01404] [INSPIRE].

[15] S. Dwivedi, V.K. Singh and A. Roy, Semiclassical limit of topological Rényi entropy in 3d Chern-Simons theory, JHEP 12 (2020) 132 [arXiv:2007.07033] [InSPIRE].

[16] V.G. Turaev and O.Y. Viro, State sum invariants of 3 manifolds and quantum $6 j$ symbols, Topology 31 (1992) 865 [INSPIRE].

[17] R. Detcherry, E. Kalfagianni and T. Yang, Turaev-Viro invariants, colored jones polynomials, and volume, Quantum Topol. 9 (2018) 775.

[18] R.M. Kashaev, The Hyperbolic volume of knots from quantum dilogarithm, Lett. Math. Phys. 39 (1997) 269 [INSPIRE].

[19] P. Calabrese and J.L. Cardy, Entanglement entropy and quantum field theory, J. Stat. Mech. 0406 (2004) P06002 [hep-th/0405152] [INSPIRE].

[20] S. Nawata, P. Ramadevi and Zodinmawia, Colored Kauffman Homology and Super-A-polynomials, JHEP 01 (2014) 126 [arXiv:1310.2240] [INSPIRE].

[21] H. Murakami and J. Murakami, The colored jones polynomials and the simplicial volume of a knot, Acta Math. 186 (2001) 85.

[22] K. Hikami, Volume conjecture and asymptotic expansion of q-series, Exper. Math. 12 (2003) 319.

[23] D. Rolfsen, Knots and links, Mathematics Lecture Series, vol. 7, Publish or Perish (1990).

[24] D. Gang, M. Romo and M. Yamazaki, All-Order Volume Conjecture for Closed 3-Manifolds from Complex Chern-Simons Theory, Commun. Math. Phys. 359 (2018) 915 [arXiv: 1704.00918] [INSPIRE]. 